\title{
Tensile fracture of an adhesive joint: the role of crack length and of material mismatch
}

\author{
F. Van Loock ${ }^{\mathrm{a}}$, M. D. Thouless ${ }^{\mathrm{b}, \mathrm{c}}$, N. A. Fleck ${ }^{\mathrm{a}}$

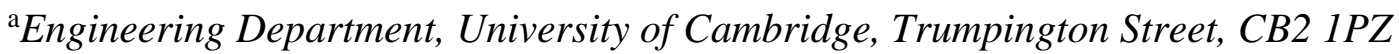 \\ Cambridge, UK \\ ${ }^{b}$ Department of Mechanical Engineering, University of Michigan, Ann Arbor, MI 48109, \\ USA \\ ${ }^{c}$ Department of Materials Science and Engineering, University of Michigan, Ann Arbor, MI \\ 48109, USA
}

\begin{abstract}
The tensile strength of an adhesive joint is predicted for a centre-cracked elastic layer, sandwiched between elastic substrates, and subjected to remote tensile stress. A tensile cohesive plastic zone, of Dugdale type, is placed at each crack tip, and the cohesive zone is characterised by a finite strength and a finite toughness. An analytical theory of the fracture strength is developed (and validated by finite element simulations). The macroscopic strength of the adhesive joint is determined as a function of the relative magnitude of crack length, layer thickness, plastic zone size, specimen width and elastic modulus mismatch between layer and substrates. Fracture maps are constructed to reveal competing regimes of behaviour. The maps span the full range of behaviour from a perfectly brittle response (with no crack tip plasticity) to full plastic collapse. When the sum of crack length and cohesive zone length is less than 0.3 times the layer height, the effect of elastic mismatch between substrate and adhesive layer has only a minor influence upon the macroscopic fracture strength. For this case, the cracked adhesive layer behaves as a centre-crack in an infinite solid made from adhesive, and a transition from toughness control to strength control occurs when the crack length is comparable to that of the cohesive zone length. Alternatively, when the sum of crack length and cohesive zone length exceeds 0.3 times the layer height, the elastic mismatch plays a major role; again there is a transition from toughness control to strength control, but it occurs at a ratio of crack length to layer thickness that depends upon both the elastic mismatch and the ratio of cohesive zone length to layer height. The study also highlights the importance of a structural length scale in the form of layer height times modulus mismatch: this scale is on the order of 1 metre when the layer height equals one millimetre and the elastic modulus of the
\end{abstract}


substrate is one thousand times that of the adhesive layer. The in-plane structural dimensions (including crack length) must exceed this structural dimension in order for a remote $K$-field to exist within the substrate. Experimental validation of the cohesive zone approach is achieved by measuring the sensitivity of fracture strength to crack length and layer height for a centrecracked strip made from cellulose acetate layer, sandwiched between aluminium alloy substrates.

Keywords: cohesive zone, adhesive layer, fracture mechanics, finite element analysis, failure maps

\section{Introduction}

There is significant interest in the use of polymer-based adhesives to bond together lightweight metallic or composite materials (such as glass fibre and carbon fibre reinforced polymers) in the aerospace, automotive, marine and wind-turbine industries (Higgins, 2000, Dillard 2010, Camanho and Tong, 2011; da Silva et al., 2018). A wide range of adhesive types (from elastomeric to epoxy) and adhesive thickness (from micron-scale to millimetre-scale) are used in joint design in order to give a joint of suitable stiffness, thickness and gap-filling capability. However, the strength of these adhesive joints is sensitive to the presence of defects, such as pores and cracks, that arise from manufacture or use. The ability to predict accurately the failure strength of adhesive joints as a function of pore or crack size is essential if engineering structures containing adhesive joints are to be manufactured in a reliable manner.

Cohesive zones are commonly used to idealise the crack tip plastic zone in metals (Barenblatt, 1962; Tvergaard and Hutchinson, 1992, 1994, 1996), crazing in polymers (Hui et al. 1992; Pandya, Ivankovic and Williams, 2000) and crack bridging in fibre composites (Schellekens and de Borst, 1996; Camanho et al., 2004; Yang and Cox 2005; Li et al., 2006). In the present study, the tensile strength of an adhesive joint is predicted for the geometry of a centre-cracked elastic layer of height $2 h$, sandwiched between elastic substrates, and subjected to remote tensile stress, as shown in Figure 1. The idealization of the adhesive by a linear, elastic solid of Young's modulus $E_{2}$, which differs from the value $E_{1}$ for the substrates, allows for the role of material mismatch $E_{2} / E_{1}$ upon the stress state (and fracture strength) to be 
explored. Initially, the centre-crack, of length $2 a$, is treated as a Griffith crack of finite crack tip toughness $\Gamma$ but with no cohesive zone present. Subsequently, a tensile cohesive plastic zone, of Dugdale type, is placed at each crack tip, and the cohesive zone is characterised by a finite strength $\hat{\sigma}$ and a finite toughness $\Gamma$. This 2-parameter description ( $\hat{\sigma}, \Gamma$ ) allows for a prediction of failure strength over a wide range of crack lengths.

It is recognized that the tensile strength $\hat{\sigma}$ and mode I toughness $\Gamma$ of an adhesive layer may depend upon the thickness of the layer (Bascom et al., 1975; Lee et al., 2004; Pardoen et al., 2005; Martiny et al., 2012) but the details of this dependence are beyond the scope of our study. The pragmatic approach adopted here is to assume that values of ( $\hat{\sigma}, \Gamma$ ) have been measured for an adhesive layer of given thickness, and the aim of this study is to explore the sensitivity of macroscopic strength to $a / h$ and $E_{1} / E_{2}$. Commonly, the measured value of $\hat{\sigma}$ is adequately approximated by the uniaxial tensile strength of the bulk adhesive (Blackman et al. 2003; Salomonsson and Andersson, 2008; Sun et al., 2008, Thouless et al., 2008; Carlberger and Stigh, 2010; Stigh et al., 2010) but it may depend upon the degree of plastic constraint (Varias et al., 1991; Tvergaard and Hutchinson, 1996; Pardoen et al., 2005). Size effects can also exist for thin adhesive layers, see, for example, Fiedler et al. (2001) and Chevalier et al. (2016).

The magnitude of $\Gamma$ will depend upon the degree of crack extension if the solid displays a pronounced $R$-curve. However, polymeric adhesive commonly display a negligible $R$-curve particularly in the form of a thin layer between substrates. This reduction in $R$-curve due to constraint has been quantified for a thin metallic sandwich layer by Tvergaard and Hutchinson (1996). Typically, the ratio $\Gamma_{\mathrm{ss}} / \Gamma_{0}$ of steady-state fracture toughness $\Gamma_{\mathrm{ss}}$ to the initiation value $\Gamma_{0}$ for polymeric adhesives in bulk form is of magnitude 1.5 for most epoxies ${ }^{1}$, acrylic, and polyurethane-based adhesives (Blackman et al., 2003; Martiny et al., 2012; Banea et al., 2014; Monteiro et al., 2015; Lopes et al. 2016; Bonaldo et al., 2018). The degree of R-curve is reduced when the adhesive is in the form of a thin layer between elastic substrates, as explained by Tvergaard and Hutchinson (1996). Consequently, the effect of an $R$-curve on crack growth is ignored in the present study.

\footnotetext{
${ }^{1}$ Rubber-modified epoxies are an exception as they may exhibit a pronounced $R$-curve in bulk form (Du et al., 1998; Imanaka et al., 2015).
} 


\subsection{The semi-infinite crack in an adhesive layer}

Consider first the asymptotic problem of an elastic adhesive layer of height $2 h$ sandwiched between two elastic substrates, and containing a semi-infinite crack, as shown in Figure 2. The crack is placed along the mid-plane of the adhesive layer. Assume that the substrate (material 1) and the adhesive (material 2) are isotropic, homogeneous and linear elastic solids. The Young's modulus and Poisson's ratio of the substrate and adhesive are $\left(E_{1}, v_{1}\right)$ and $\left(E_{2}, v_{2}\right)$, respectively. We consider a general, plane problem, where $\bar{E}$ and $\bar{v}$ denote $E$ and $v$ in plane stress, but $\bar{E}=E /\left(1-v^{2}\right)$ and $\bar{v}=v /(1-v)$ in plane strain. This crack problem has been considered before by Fleck et al. (1991) and by Ryvkin (2000) using Fourier transforms, but the findings below were not presented in these previous studies.

In the introductory problem under consideration, the adhesive joint is subjected to a remote mode-I $K$ field of magnitude $K^{\infty}$. The normal stress component $\sigma_{y y}$, perpendicular to the crack plane and directly ahead of the crack tip, has been calculated by finite element (FE) analysis, for selected values of modulus-mismatch ratio $\bar{E}_{2} / \bar{E}_{1}$ and $\overline{v_{1}}=\overline{v_{2}}=3 / 7$; the details of this routine calculation are reported in Appendix A. The normalized stress component, $\sigma_{y y} \sqrt{h} / K^{\infty}$, is plotted in Figure 3a as a function of normalized distance from the crack tip, $x / h$, for the representative case $\bar{E}_{2} / \bar{E}_{1}=10^{-2}$.

Path-independence of the $J$-integral (Rice, 1968) implies that the remote $K^{\infty}$-field and the local $K^{\text {tip }}$-field at the crack tip are related by (Trantina 1972, Wang et al., 1978; Fleck et al., 1991)

$$
K^{\mathrm{tip}}=\left(\frac{\bar{E}_{2}}{\bar{E}_{1}}\right)^{\frac{1}{2}} K^{\infty} .
$$

Hence, as can be seen in Figure 3a, the stress at the crack tip within the adhesive layer is shielded by the presence of stiffer substrate material. Adjacent to the crack tip, the stresses are given by the leading term of the William's singularity analysis (Williams, 1957), with $\sigma_{y y}$ given by 


$$
\frac{\sigma_{y y} \sqrt{h}}{K^{\infty}}=\left(\frac{1}{2 \pi} \frac{\bar{E}_{2}}{\bar{E}_{1}} \frac{h}{x}\right)^{\frac{1}{2}} .
$$

Consistent with the requirement (1), the stress component $\sigma_{y y}$ remote from the crack tip scales as

$$
\frac{\sigma_{y y} \sqrt{h}}{K^{\infty}}=\left(\frac{1}{2 \pi} \frac{h}{x}\right)^{\frac{1}{2}} .
$$

Equations (2) and (3) provide asymptotes to the stress distribution ahead of the crack tip, as plotted in Figure 3a. The stress follows the local $K^{\text {tip }}$ field close to the crack tip provided $x / h$ is less than a transition value designated by $(x / h)_{\mathrm{T} 1}$. Alternatively, at sufficiently large $x / h$, above a second transition value $(x / h)_{\mathrm{T} 2}$, the stress state is given by the remote $K^{\infty}$ field. Relations (2) and (3) imply that

$$
\left(\frac{x}{h}\right)_{\mathrm{T} 2}=\frac{\bar{E}_{1}}{\bar{E}_{2}}\left(\frac{x}{h}\right)_{\mathrm{T} 1} .
$$

The stress distribution is plotted as a function of $x / h$ along the crack plane for selected values of modulus-mismatch ratio in the range $\bar{E}_{2} / \bar{E}_{1}=1$ to $\bar{E}_{2} / \bar{E}_{1}=10^{-3}$ in Figure 3b. The tensile stress has a plateau value for $x / h$ values intermediate between the two transition values $(x / h)_{\mathrm{T} 1}$ and $(x / h)_{\mathrm{T} 2}$. As the modulus mismatch becomes more extreme, the plateau stress tends to the asymptotic limit of

$$
\frac{\sigma_{y y} \sqrt{h}}{K^{\text {tip }}}=\left(1-\bar{v}_{2}^{2}\right)^{-1 / 2},
$$

which is the solution for a semi-infinite crack in an adhesive layer between two rigid substrates and subjected to a uniform opening displacement (Knauss, 1966; Rice, 1967; Wang, 1997).

Note from Figure $3 \mathrm{~b}$ that the value of $(x / h)_{\mathrm{T} 1}$ equals approximately 0.2 independent of the magnitude of $\bar{E}_{2} / \bar{E}_{1}$ over the range considered ${ }^{2}$. Consequently, relation (4) reduces to

\footnotetext{
${ }^{2}$ Ryvkin (2000) adopted a more severe criterion for the definition of $(x / h)_{\mathrm{T} 1}$ by the location at which the value of $\sigma_{y y}$ deviated by $5 \%$ from its asymptotic value (2), and thereby concluded that $(x / h)_{\mathrm{T} 1}=0.03$ for $\bar{E}_{2} / \bar{E}_{1}<0.1$.)
} 
$(x / h)_{\mathrm{T} 2}=0.2 \bar{E}_{1} / \bar{E}_{2}$, with the immediate implication that a remote $K^{\infty}$ field can only exist in a specimen of characteristic in-plane dimensions (such as crack length, height and ligament width) that significantly exceed the value of $0.2 h \bar{E}_{1} / \bar{E}_{2}$. Assume that an in-plane dimension

on the order of $h \bar{E}_{1} / \bar{E}_{2}$ is required to meet this condition. This places a severe restriction on the relevance of a remote stress intensity factor for thick polymeric adhesive joints between metallic or composite substrates. For example, consider an epoxy adhesive of thickness $1 \mathrm{~mm}$ and Young's modulus 1 GPa sandwiched between steel substrates of modulus $210 \mathrm{GPa}$. Then, an in-plane structural dimension of $h \bar{E}_{1} / \bar{E}_{2}=210 \mathrm{~mm}$ is required in order for a remote $K$-field to exist. This requirement is significantly more restrictive for the choice of a thick elastomeric adhesive between steel substrates (as used in shipbuilding) such that $h=10 \mathrm{~mm}$ and $\bar{E}_{2}=0.1$ GPa; the minimum in-plane dimension then becomes $21 \mathrm{~m}$. For such applications, it is necessary to consider a prototypical specimen of finite crack length and subjected to a remote stress, such as the centre-cracked sandwich panel shown in Figure 1, and recognize the fact that a remote $K$-field may not exist for this specimen. This is the main geometry under consideration in the present paper.

\section{Scope of study}

The aim of this study is to determine the sensitivity of the tensile strength of an adhesive joint to crack length, thickness of adhesive layer, modulus-mismatch ratio, and to the toughness and cohesive strength of the adhesive. Mode I loading of the crack within the adhesive layer is considered, and the crack is either a classical Griffith crack (with a finite value of tip toughness but absent a cohesive zone) or the crack is endowed with a tensile cohesive zone of both finite strength and finite toughness. An experimental study on a centre-cracked cellulose acetate strip, sandwiched between aluminium alloy substrates, is used to validate and illustrate the theory.

\section{Theory}

Consider the finite sandwich joint shown in Figure 1. The joint consists of an adhesive layer of height $2 h$ and width $2 W$ sandwiched between two substrates, each of length $L$. A through-thickness centre-crack of length $2 a$ lies parallel to the interface at mid-height of the adhesive layer. The substrate is identified as material 1 , and the adhesive is identified as 
material 2, each being isotropic, homogeneous and linear-elastic, with elastic properties, $\bar{E}_{1}$, $\overline{v_{1}}$ and $\bar{E}_{2}, \overline{v_{2}}$, as defined above. The sandwich layer is loaded by a remote tensile stress $\sigma^{\infty}$ parallel to the $y$-axis. It is assumed that the adhesive has a crack-tip cohesive zone that obeys a tensile traction versus separation law of the form shown in Figure 1, with a finite cohesive strength $\hat{\sigma}$ and finite toughness. The presence of the cohesive zone leads to the existence of a material length scale $l_{\mathrm{s}}$ of magnitude

$$
l_{\mathrm{S}}=\frac{\bar{E}_{2} \Gamma}{\pi \hat{\sigma}^{2}}
$$

following Tvergaard and Hutchinson (1992). The macroscopic strength of the sandwich joint $\sigma_{\mathrm{f}}^{\infty}$ is a function of normalized crack length $a / h$, modulus-mismatch ratio $\bar{E}_{2} / \bar{E}_{1}$, and $l_{\mathrm{s}} / h$, and can be written in non-dimensional form as ${ }^{3}$

$$
\bar{\sigma}=\frac{\sigma_{\mathrm{f}}^{\infty} \sqrt{h}}{\sqrt{\bar{E}_{2} \Gamma}}=f\left(\frac{a}{h}, \frac{W}{h}, \frac{\bar{E}_{2}}{\bar{E}_{1}}, \frac{l_{\mathrm{s}}}{h}, \bar{v}_{1}, \bar{v}_{2}\right) .
$$

The role of Poisson's ratio is minor and is neglected in this study. However, the sensitivity of joint strength to the other non-dimensional groups is explored in detail. First, we will consider the limiting case of a Griffith crack in an elastic layer of finite toughness but unbounded cohesive strength such that $l_{\mathrm{S}} / h \rightarrow 0$. Then, we take into account the existence of a finite value of cohesive strength $\hat{\sigma}$.

\subsection{Griffith crack in an elastic layer}

We begin by considering the two extreme cases of a very short crack and a very long crack in an elastic, perfectly brittle adhesive layer (of unbounded cohesive strength $\hat{\sigma}$ ), and then consider the more complex case of an intermediate crack length. The terms 'short', 'long' and 'intermediate’ are made precise below.

\section{Asymptotic analysis for a very short or very long crack}

In the limit of a very short crack length $a / h<<1$, the presence of the substrate can be ignored, and the crack tip stress intensity factor is given by

\footnotetext{
${ }^{3}$ Describing the elastic constants by Dundurs' parameters would reduce the number of terms by one. However, the effects of Poisson's ratio are not a focus of this study, and so this rationalization is not employed.
} 


$$
K^{\text {tip }}=\sigma^{\infty} \sqrt{\pi a} .
$$

Crack growth occurs when the energy release rate $G$ attains the toughness $\Gamma$ of the adhesive, such that

$$
\mathrm{G}=\frac{\left(K^{\mathrm{tip}}\right)^{2}}{\bar{E}_{2}}=\Gamma,
$$

and the macroscopic fracture strength is then given by

$$
\bar{\sigma}=\frac{\sigma_{\mathrm{f}}^{\infty} \sqrt{h}}{\sqrt{\bar{E}_{2} \Gamma}}=\left(\frac{h}{\pi a}\right)^{\frac{1}{2}} .
$$

Alternatively, for a very long crack, $a / h>>1$, the presence of the adhesive layer can be ignored. Provided that $a / W<<1$, the appropriate stress-intensity factor is given by

$$
K^{\infty}=\sigma^{\infty} \sqrt{\pi a}
$$

Then, from (1), the failure strength of the joint is

$$
\bar{\sigma}=\frac{\sigma_{\mathrm{f}}^{\infty} \sqrt{h}}{\sqrt{\bar{E}_{2} \Gamma}}=\left(\frac{\bar{E}_{1}}{\bar{E}_{2}} \frac{h}{\pi a}\right)^{\frac{1}{2}} .
$$

General analysis for an intermediate length of crack

We proceed to develop analytical expressions for $\bar{\sigma}$ for a crack of intermediate length with respect to both the adhesive thickness and width of the joint. As can be seen in the Appendix B, a series of FE results confirmed the accuracy of the analysis presented below. Consider the joint shown on the left of Figure 4, and write $\Delta u$ as the extra displacement of the ends of the specimen due to the presence of a crack of length $2 a$ and an end load $P$ per unit thickness. Thus, $\Delta u$ equals $P \Delta C$ where $\Delta C(a) \equiv C(a)-C(0)$ is the extra compliance due to the presence of the crack. Note that (Tada et al., 2000):

$$
\mathrm{G}=\frac{P^{2}}{4} \frac{\partial(\Delta C)}{\partial a}
$$

and, for a specimen of infinite height, $C$ is unbounded but $\Delta C$ is finite. Now proceed to idealise the adhesive joint problem on the left of Figure 4 by the summation of two problems on the right-hand side, case (1) and case (2), such that 


$$
\frac{\partial(\Delta C)}{\partial a}=\frac{\partial\left(\Delta C^{(1)}\right)}{\partial a}+\frac{\partial\left(\Delta C^{(2)}\right)}{\partial a}
$$

Case 1 neglects the presence of the adhesive layer in the determination of the additional compliance due to the presence of the crack. Case 2 is an approximate analysis for the additional compliance due to the presence of the crack when a strip of height $2 h$ and made from material 1 is replaced by a strip of material 2 . Thus, in case 2, the substrate is treated as rigid and the replacement strip is of effective modulus $\hat{E}$ such that:

$$
\frac{1}{\hat{E}}=\frac{1-\bar{v}_{2}^{2}}{\bar{E}_{2}}-\frac{1-\bar{v}_{1}^{2}}{\bar{E}_{1}}
$$

The constraint factors $\left(1-\bar{v}_{1}^{2}\right)$ and $\left(1-{\overline{v_{2}}}^{2}\right)$ arise from the fact that the strain component in the replacement layer vanishes in the tangential direction.

We seek expressions for $\partial\left(\Delta C^{(1)}\right) / \partial a$ and $\partial\left(\Delta C^{(2)}\right) / \partial a$. From Tada et al. (2000), $\partial\left(\Delta C^{(1)}\right) / \partial a$ satisfies:

$$
\frac{\partial\left(\Delta C^{(1)}\right)}{\partial a}=\frac{\pi a F^{2}}{W^{2} \bar{E}_{1}}
$$

where the finite width correction factor $F(a / W)$ has already been determined (Federsen, 1966; Tada et al., 2000):

$$
F=\left[1-0.025\left(\frac{a}{W}\right)^{2}+0.06\left(\frac{a}{W}\right)^{4}\right]\left[\sec \left(\frac{\pi}{2} \frac{a}{W}\right)\right]^{\frac{1}{2}}
$$

For case (2), the net section stress is

$$
\sigma^{\text {net }}=\frac{P}{2 W}\left(1-\frac{a}{W}\right)^{-1}
$$

and the specimen compliance is approximately given by

$$
\Delta C^{(2)}(a)=C^{(2)}(a)=\frac{h}{W \hat{E}(1-(a / W))},
$$


Now, make use of the Irwin relationship, $K^{\text {tip }}=\left(\bar{E}_{2} G\right)^{\frac{1}{2}}$ and relation (13) to obtain

$$
K^{\mathrm{tip}}=\frac{P}{2}\left(\bar{E}_{2} \frac{\partial(\Delta C)}{\partial a}\right)^{\frac{1}{2}}
$$

The normalized strength $\bar{\sigma}$, as defined in (7), is related to $P$ and $K^{\text {tip }}$ by

$$
\bar{\sigma}=\frac{\sigma_{\mathrm{f}}^{\infty} \sqrt{h}}{\sqrt{\bar{E}_{2} \Gamma}}=\frac{P}{2 W} \frac{\sqrt{h}}{K^{\mathrm{tip}}} .
$$

Upon making use of (14), (16), (18) and (20), we then obtain the general formula

$$
\bar{\sigma}=\left[\pi \frac{\bar{E}_{2}}{\bar{E}_{1}} \frac{a}{h} F^{2}+\left(\left(1-\bar{v}_{2}^{2}\right)-\left(1-\bar{v}_{1}^{2}\right) \frac{\bar{E}_{2}}{\bar{E}_{1}}\right)\left(1-\frac{a}{W}\right)^{-2}\right]^{-1 / 2} .
$$

For $\bar{v}=\overline{v_{1}}=\bar{v}_{2}$, this relation reduces to

$$
\bar{\sigma}=\left[\pi \frac{\bar{E}_{2}}{\bar{E}_{1}} \frac{a}{h} F^{2}+\left(1-\bar{v}^{2}\right)\left(1-\frac{\bar{E}_{2}}{\bar{E}_{1}}\right)\left(1-\frac{a}{W}\right)^{-2}\right]^{-1 / 2} .
$$

Note that (23) implies a plateau value in the strength for intermediate values of $a / h$, and for $\bar{E}_{2} / \bar{E}_{1}<<1$ this reduces to the limit

$$
\bar{\sigma}=\left(1-\bar{v}_{2}^{2}\right)^{-1 / 2},
$$

consistent with (5) above, upon noting $K^{\text {tip }}=\sqrt{\bar{E}_{2} \Gamma}$ and $\sigma_{y y}=\sigma_{\mathrm{f}}^{\infty}$. For very small cracks, the small-crack asymptote (10) becomes valid. The transition between these two regimes is obtained by equating the strengths from expressions (10) and (24), thereby giving a crack length of

$$
\frac{a}{h}=\frac{1-\bar{v}_{2}^{2}}{\pi}
$$

The expressions (10) and (23) for $\bar{\sigma}$ are plotted as a function of $a / h$ in Figure 5a for the case $h / W=0$ and for selected values of $\bar{E}_{2} / \bar{E}_{1}$ in the range of 0 to 1 . The short crack asymptote (10) exists in the region as identified as regime B in this plot, whereas the case of intermediate to long cracks is termed regime C. (A more precise definition of these regimes will be made 
clear below). The long-crack asymptotes of (12) are included in the plot of Figure 5a. The fracture strength decreases with increasing $\bar{E}_{2} / \bar{E}_{1}$ in regime C, but is independent of the magnitude of $\bar{E}_{2} / \bar{E}_{1}$ in the short crack regime B. The sensitivity of the strength $\bar{\sigma}$ to crack length, for a modulus-mismatch ratio of $\bar{E}_{2} / \bar{E}_{1}=10^{-2}$ and for selected values of $h / W$ ranging from 0 to 0.1 , is given in Figure 5b. As the crack length $a$ approaches $W$ the strength $\bar{\sigma}$ drops sharply: this is consistent with the usual form of the $K$-calibration for a finite specimen.

\subsection{Effect of plasticity at the crack tip: cohesive zone model}

\section{Asymptotic analysis for a very short crack}

We proceed to explore the effect of a finite length of cohesive zone at the crack tip on the strength of the joint as depicted in Figure 1. We emphasize that the cohesive zone is of uniform strength $\hat{\sigma}$ and toughness $\Gamma$, and the material length scale $l_{\mathrm{S}}$ is a derived material property via (6). In general, $l_{\mathrm{S}}$ is not equal to the length $c$ of cohesive zone: this is only the case for a semi-infinite crack in a homogeneous, infinite solid made from the adhesive. For example, consider the case of a short crack of length $2 a$ in a homogeneous, infinite solid, such that $l_{\mathrm{S}}>$ $a$. Then, at failure, the remote stress $\sigma_{\mathrm{f}}^{\infty}$ is slightly less than $\hat{\sigma}$ but the cohesive zone size $c$ exceeds both $a$ and $l_{\mathrm{S}}$.

Consider first the case for which $a+l_{\mathrm{S}}<<h$. Then, the crack and cohesive zone exist within a much larger layer of adhesive, and the joint strength can be predicted by ignoring the presence of the substrate. We can then make direct use of the strip yield model of Dugdale (1960) and Barenblatt (1962), as is appropriate for the assumed traction-separation relationship defined in Figure 1. In the Dugdale model, the crack-tip opening displacement $\delta^{\text {tip }}$ for a through crack of length $2 a$ in a linear-elastic, infinite sheet under a remote uniaxial tensile stress $\sigma^{\infty}$ is given by

$$
\delta^{\mathrm{tip}}=\frac{8 \hat{\sigma} a}{\pi \bar{E}_{2}} \ln \left[\sec \left(\frac{\pi \sigma^{\infty}}{2 \hat{\sigma}}\right)\right] .
$$


Now recognize that $\Gamma=\hat{\sigma} \delta_{\mathrm{C}}$, where $\delta_{\mathrm{C}}$ is the value of $\delta^{\text {tip }}$ at $\sigma^{\infty}=\sigma_{\mathrm{f}}^{\infty}$; then (26) can be reexpressed as

$$
\frac{l_{\mathrm{S}}}{h}=\frac{\bar{E}_{2} \delta_{\mathrm{C}}}{\pi \hat{\sigma} h}=\frac{8}{\pi^{2}} \frac{a}{h} \ln \left[\sec \left(\frac{\pi \sigma_{\mathrm{f}}^{\infty}}{2 \hat{\sigma}}\right)\right]
$$

and is valid provided that $a+l_{\mathrm{s}}<<h$ and $h / W \leq 1$. This equation is an implicit relation for $\bar{\sigma}$ as a function of $a / h$, upon noting the direct connection between $\bar{\sigma}$ and $\sigma_{\mathrm{f}}^{\infty} / \hat{\sigma}$ via the definition (6) for $l_{\mathrm{s}}$ :

$$
\bar{\sigma} \equiv \frac{\sigma_{\mathrm{f}}^{\infty} \sqrt{h}}{\sqrt{\bar{E}_{2} \Gamma}}=\frac{\sigma_{\mathrm{f}}^{\infty}}{\hat{\sigma}} \frac{\hat{\sigma} \sqrt{h}}{\sqrt{\bar{E}_{2} \Gamma}}=\frac{\sigma_{\mathrm{f}}^{\infty}}{\hat{\sigma}}\left(\frac{h}{\pi l_{s}}\right)^{1 / 2} .
$$

\section{General analysis for an intermediate crack length}

We proceed to address the case where the combined length of $a$ and $l_{\mathrm{S}}$ are on the order of, or larger than, $h$. Again, an approximate analytical analysis is performed for the cohesive zone problem, as stated in the left hand side of Figure 6. In order to confirm the analytical results of this section, we conducted additional FE calculations to compute the dependence of strength upon geometry and modulus mismatch ratio. The numerical results are presented in Appendix B and confirm the accuracy of the analyses presented here.

The analytical approach assumes that the crack tip displacement for the full problem (as given in the left hand side of Figure 6) is adequately given by the superposition of the crack tip opening displacement for problems (1) and (2) as stated on the right hand side of Figure 6:

$$
\delta^{\mathrm{tip}}=\delta^{(1)}+\delta^{(2)} .
$$

Here, $\delta^{(1)}$ is the crack-tip opening displacement for a crack of length $2 a$ in a linear-elastic sheet of infinite width of material 1 under a remote tensile stress of $\sigma^{\infty}$ (see case 1 of Figure 6), and $\delta^{(2)}$ is the crack-tip opening displacement for a crack of length $2 a$ in a linear-elastic sandwich layer with Young's modulus equal to $\hat{E}$, defined earlier in (15), and clamped between two rigid substrates of finite width subjected to a remote tensile stress $\sigma^{\infty}$ (see case 2 of Figure 6). This approximation is exact in the limit of $\bar{E}_{2} / \bar{E}_{1}=0$ and for the homogeneous case $\bar{E}_{2} / \bar{E}_{1}=1$. 
Now make use of (26) to write the crack-tip opening displacement of case 1 as

$$
\delta^{(1)}=\frac{8 \hat{\sigma} a}{\pi \bar{E}_{1}} \ln \left[\sec \left(\frac{\pi \sigma^{\infty}}{2 \hat{\sigma}}\right)\right] .
$$

For case 2, the value of the $J$-integral taken around the crack tip is given by

$$
J^{\text {tip }}=\hat{\sigma} \delta^{(2)} .
$$

However, for a linear-elastic system the value of the $J$-integral taken around a remote contour $J^{\infty}$ equals the energy-release rate, and can be deduced from the expression for the derivative of the compliance as given by (19), such that

$$
J^{\infty}=\frac{h \sigma^{\infty^{2}}}{\hat{E}}\left(1-\frac{a}{W}\right)^{-2} .
$$

Upon invoking the path-independence of the $J$-integral, (31) and (32) can be combined to give

$$
\delta^{(2)}=\frac{h}{\hat{E}} \frac{\sigma^{\infty^{2}}}{\hat{\sigma}}\left(1-\frac{a}{W}\right)^{-2} .
$$

The relation (29) becomes, via (30) and (33),

$$
\frac{\delta^{\mathrm{tip}}}{h}=\frac{8 \hat{\sigma}}{\pi \bar{E}_{1}} \frac{a}{h} \ln \left[\sec \left(\frac{\pi \sigma^{\infty}}{2 \hat{\sigma}}\right)\right]+\frac{\sigma^{\infty^{2}}}{\hat{E} \hat{\sigma}}\left(1-\frac{a}{W}\right)^{-2} .
$$

Upon recalling the definition of $l_{\mathrm{S}}$ in (6), we can rephrase $l_{\mathrm{S}}$ as $l_{\mathrm{S}} \equiv \delta_{\mathrm{C}} \bar{E}_{2} / \pi \hat{\sigma}$, where $\delta_{\mathrm{C}}$ is the critical crack-tip opening displacement. At fracture, $\sigma^{\infty}=\sigma_{\mathrm{f}}^{\infty}$ and $\delta^{\text {tip }}=\delta_{\mathrm{C}}$, thereby giving our main result

$$
\frac{l_{\mathrm{S}}}{h}=\frac{8}{\pi^{2}} \frac{\bar{E}_{2}}{\bar{E}_{1}} \frac{a}{h} \ln \left[\sec \left(\frac{\pi \sigma_{\mathrm{f}}^{\infty}}{2 \hat{\sigma}}\right)\right]+\frac{1}{\pi}\left(\frac{\sigma_{\mathrm{f}}^{\infty}}{\hat{\sigma}}\right)^{2}\left[\left(1-\bar{v}_{2}^{2}\right)-\left(1-\bar{v}_{1}^{2}\right) \frac{\bar{E}_{2}}{\bar{E}_{1}}\right]\left(1-\frac{a}{W}\right)^{-2} .
$$

For the case $\bar{v}=\bar{v}_{1}=\bar{v}_{2}$, this relation reduces to

$$
\frac{l_{\mathrm{S}}}{h}=\frac{8}{\pi^{2}} \frac{\bar{E}_{2}}{\bar{E}_{1}} \frac{a}{h} \ln \left[\sec \left(\frac{\pi \sigma_{\mathrm{f}}^{\infty}}{2 \hat{\sigma}}\right)\right]+\frac{1-\bar{v}^{2}}{\pi}\left(\frac{\sigma_{\mathrm{f}}^{\infty}}{\hat{\sigma}}\right)^{2}\left(1-\frac{\bar{E}_{2}}{\bar{E}_{1}}\right)\left(1-\frac{a}{W}\right)^{-2} .
$$

We note in passing that, in the limit of $\sigma_{\mathrm{f}}^{\infty} / \hat{\sigma} \rightarrow 0$, expression (36) reduces to

$$
\frac{1}{\bar{\sigma}^{2}}=\frac{\bar{E}_{2} \Gamma}{\left(\sigma_{\mathrm{f}}^{\infty}\right)^{2} h}=\pi \frac{\bar{E}_{2}}{\bar{E}_{1}} \frac{a}{h}+\left(1-\bar{v}^{2}\right)\left(1-\frac{\bar{E}_{2}}{\bar{E}_{1}}\right)\left(1-\frac{a}{W}\right)^{-2} .
$$


which is in close alignment with (23) upon re-writing (23) as

$$
\frac{1}{\bar{\sigma}^{2}}=\pi \frac{\bar{E}_{2}}{\bar{E}_{1}} \frac{a}{h} F^{2}+\left(1-\bar{v}^{2}\right)\left(1-\frac{\bar{E}_{2}}{\bar{E}_{1}}\right)\left(1-\frac{a}{W}\right)^{-2}
$$

The expressions (37) and (38) differ only by the factor $F^{2}$ in the first term on the right hand side of (38). Note that $F$, as defined in (17), is close to unity for small values of $a / W$. For example, $F(a / W=0)=1$ and $F(a / W=0.3)=1.06$. Further, the first term on the right hand side of (37) and (38) is negligible in comparison to the second term for sufficiently small values of $\bar{E}_{2} / \bar{E}_{1}$.

\subsection{Regimes of behaviour}

There are four regimes of behaviour for the joint problem as defined in Figure 1. The four regimes can be plotted on a map with axes $a / h$ and $l_{\mathrm{S}} / h$ as follows. First, there is a broad division into whether the behaviour can be described by an asymptotic limit for which the effect of the substrate can be ignored. This condition is approximated by the geometric relation

$$
\left(l_{\mathrm{S}}+a\right)<0.3 h
$$

It is illustrated in the $l_{\mathrm{S}} / \mathrm{h}$ versus $a / h$ failure map as a solid transition line separating regions A and B from regions C and D, see Figure 7. Within the adhesive-governed regime that satisfies (37), there is a sub-division between toughness-controlled fracture and strength-controlled fracture, defined as follows. We adopt the criterion that strength-controlled fracture occurs when $\sigma_{\mathrm{f}}^{\infty} / \hat{\sigma}>0.99$, which implies from (27) that

$$
\frac{l_{\mathrm{S}}}{a}>3.37
$$

This criterion has been added to Figure 7 to distinguish between regime A, where fracture is controlled by strength, and regime B where fracture is controlled by toughness. We emphasize that this boundary is independent of the value of $\bar{E}_{2} / \bar{E}_{1}$.

Now consider the geometries that satisfy $\left(l_{\mathrm{S}}+a\right)>0.3 h$ such that the presence of the substrate needs to be accounted for. Again there exists two sub-regions, labelled $\mathrm{C}$ for the regime of toughness-control and regime $\mathrm{D}$ for strength-control. It is convenient to identify the boundary C/D between toughness-controlled fracture and strength-controlled fracture in the 
substrate-governed regime again by the criterion $\sigma_{\mathrm{f}}^{\infty} / \hat{\sigma}=0.99$. The resulting trajectory of the C/D boundary in $\left(l_{\mathrm{s}} / \mathrm{h}, a / h\right)$ space follows directly from (36) and is expressed by

$$
\frac{l_{\mathrm{S}}}{h}=3.37 \frac{\bar{E}_{2}}{\bar{E}_{1}} \frac{a}{h}+0.31\left(1-\bar{v}^{2}\right)\left(1-\frac{\bar{E}_{2}}{\bar{E}_{1}}\right)\left(1-\frac{a}{W}\right)^{-2},
$$

for $\bar{v}_{1}=\bar{v}_{2}=\bar{v}$. This boundary is plotted in Figure 7 for $\overline{\boldsymbol{v}}=3 / 7, h / W=0$ and for $\bar{E}_{2} / \bar{E}_{1}$ equal to zero or 0.01. Sketches are included in Figure 7 to illustrate the relative magnitude of the length scales $(c / h, a / h)$ in regimes A to $\mathrm{D}$, where c is the length of the cohesive zone at fracture, and is, in general, different from the material length scale $l_{\mathrm{s}}$. Contours of strength $\sigma_{\mathrm{f}}^{\infty} / \hat{\sigma}=(0.1,0.35,0.99)$ have also been added to Figure 7 by making use of (27) and (36).

It is instructive to cross-plot $\sigma_{\mathrm{f}}^{\infty} / \hat{\sigma}$ as a function of $a / h$ in Figure 8a for selected values of $l_{S} / h$ and for $\bar{E}_{2} / \bar{E}_{1}=10^{-2}, \overline{\boldsymbol{v}}=3 / 7$, and $h / W=0$. The plots have again been constructed by making use of (27) and (36). Predictions for $\sigma_{\mathrm{f}}^{\infty} / \hat{\sigma}$ are included for the limit $\bar{E}_{2} / \bar{E}_{1}=0$ (shown as dotted lines). Recall that $\sigma_{\mathrm{f}}^{\infty} / \hat{\sigma}$ can be re-written in terms of $\bar{\sigma}=\sigma_{\mathrm{f}}^{\infty} \sqrt{h} / \sqrt{\bar{E}_{2} \Gamma}$ and of $l_{\mathrm{S}} / \mathrm{h}$ by making use of the identity (28). Also, in the limit of an elastic, ideally brittle Griffith crack, $\hat{\sigma}$ is unbounded whereas $\sigma_{\mathrm{f}}^{\infty} / \hat{\sigma}$ and $l_{\mathrm{s}} / h$ both vanish. The non-dimensional strength $\bar{\sigma}$ remains finite in this limit. Thus $\bar{\sigma}$ has been plotted as a function of $a / h$ in Figure $8 \mathrm{~b}$ in order to make contact with the results for the Griffith crack, recall Figure 5.

Now re-consider Figure 7, and focus on the transitions from regime to regime with increasing $a / h$, for 3 selected values of $l_{\mathrm{S}} / h=10^{-3}, 0.1$ and 1 . First, for $l_{\mathrm{S}} / \mathrm{h}=10^{-3}$, the active fracture mode switches from the adhesive-governed regime B to the substrate-governed regime $\mathrm{C}$ with increasing $a / h$. Both regimes B and C are toughness-controlled. The plot of $\bar{\sigma}$ versus $a / h$ for $\bar{E}_{2} / \bar{E}_{1}=0$ in Figure $8 \mathrm{~b}$ is indistinguishable from that for $\bar{E}_{2} / \bar{E}_{1}=10^{-2}$ in regime $\mathrm{B}$, but the predictions become sensitive to the value of $\bar{E}_{2} / \bar{E}_{1}$ with increasing $a / h$ in regime $\mathrm{C}$. 
Second, assume a small length of plastic zone, as parameterised by $l_{\mathrm{S}} / h=0.1$, in the map of Figure 7; with increasing $a / h$ there is a transition from strength-control, regime A, to toughness-control, regimes $\mathrm{B}$ and then $\mathrm{C}$. In regime $\mathrm{A}, \sigma_{\mathrm{f}}^{\infty} / \hat{\sigma}$ equals unity (see Figure $8 \mathrm{a}$ ). Then, for $0.03<a / h<0.25$ regime $B$ is active, such that the crack and its cohesive zone are shorter than the adhesive height $\left(a+l_{\mathrm{S}}<0.3 h\right)$ and $\sigma_{\mathrm{f}}^{\infty} / \hat{\sigma}$ drops with increasing $a / h$, see Figures $8 \mathrm{a}$ or $8 \mathrm{~b}$. At longer crack lengths, $a / h>0.25$, regime $C$ is entered and the strength $\sigma_{\mathrm{f}}^{\infty} / \hat{\sigma}$ is below the yield value of unity and independent of $a / h$ for $\bar{E}_{2} / \bar{E}_{1}=0$, see Figure 8a. In contrast, for $\bar{E}_{2} / \bar{E}_{1}=10^{-2}, \sigma_{\mathrm{f}}^{\infty} / \hat{\sigma}$ decreases with increasing $a / h$ due to the fact that the crack (and cohesive zone) are embedded within the outer K-field of the substrate, and the long crack asymptote (12) is approached.

Third, consider the case $l_{\mathrm{S}} / h=1$ in the map of Figure 7 . The response is in regime $\mathrm{D}$ such that $\sigma_{\mathrm{f}}^{\infty} / \hat{\sigma}=1$ for the choice $\bar{E}_{2} / \bar{E}_{1}=0$ and all $a / h$. Alternatively, for $\bar{E}_{2} / \bar{E}_{1}=10^{-2}$, the response switches from strength control (regime D) to toughness-control (regime C) at $a / h$ $=15$, and for crack lengths that exceed this transition value $\sigma_{\mathrm{f}}^{\infty} / \hat{\sigma}$ drops with crack extension: the crack (and cohesive zone) are embedded within the outer $K$-field of the substrate in a similar manner to that discussed for $l_{\mathrm{S}} / h=0.1$.

The transition from strength control (regime D) to toughness control (regime C) in the fracture map of Figure 7 occurs at a value of $a / h$ that depends upon both the modulus mismatch $\bar{E}_{2} / \bar{E}_{1}$ and $l_{\mathrm{S}} / h$. This C/D boundary is re-plotted in Figure 9 using axes of $\left(\bar{E}_{2} / \bar{E}_{1}, l_{\mathrm{S}} / h\right)$ and contours of $a / h$, for the choice $\overline{\boldsymbol{v}}=3 / 7$ and $h / W=0$. The map has the following useful interpretation: for any given adhesive/substrate combination, the values of $\left(\bar{E}_{2} / \bar{E}_{1}, l_{\mathrm{S}} / h\right)$ are known and this combination of properties and geometry (via $\left.h\right)$ can be plotted as a point on Figure 9. Thereby, the transition value of $a / h$ from strength to toughness controlled fracture can be identified. It is notable that the transition value of $a / h$ equals 0.1 for a very wide range of modulus mismatch $\bar{E}_{2} / \bar{E}_{1}$ for the choice $l_{\mathrm{S}} / \mathrm{h}=0.3$.

It remains to provide experimental support for the above theory, and in particular to confirm the existence of the competing failure regimes of Figure 7 . This is now addressed in the following section for the choice of an 'adhesive layer' made from cellulose acetate and 
substrates made from an aluminium alloy. The measured value of $l_{\mathrm{s}}$ equals $1 \mathrm{~mm}$ for the cellulose acetate, and two values of layer thickness are employed, $h=1 \mathrm{~mm}$ and $5 \mathrm{~mm}$. Thus, $l_{\mathrm{S}} / h$ equals 0.2 and 1 , along with $\bar{E}_{2} / \bar{E}_{1}=0.09$; these values have been added to the map of Figure 9, and values of crack length $a / h$ are employed to ensure that failure is by plastic collapse within regime D (sufficiently small $a / h$ ) or within regime C (toughness-controlled at a sufficiently large $a / h$ ) for the choice of $l_{\mathrm{S}} / h=1$. The details are as follows.

\section{Tensile strength of a cellulose acetate-aluminum sandwich layer}

\subsection{Test method}

Consider a centre-cracked sandwich specimen, with a cellulose acetate strip sandwiched between aluminum substrates as shown in Figure 10. The sandwich layer comprises a cellulose acetate strip ${ }^{4}$ of thickness $t=40 \mu \mathrm{m}$ adhered to two aluminum alloy substrates ${ }^{5}$, and of identical thickness to that of the cellulose acetate strip. In turn, the aluminium alloy substrates are adhered to aluminium alloy extension sheets of thickness $1.5 \mathrm{~mm}$, which are loaded in tension by the loading pins of a screw-driven test machine (see Figure 10). The longitudinal direction of the cellulose acetate tape is aligned with a centre-crack of length $2 a$ and with the $x$-axis, as shown in Figure 10 .

The tensile failure strength $\sigma_{\mathrm{f}}^{\infty}$ of the sandwich layer was measured as a function of crack length $2 a$. The remote tensile stress was deduced from the applied load as

$$
\sigma^{\infty}=\frac{P}{2 W t} .
$$

Tests were performed for $h=1 \mathrm{~mm}$ and $5 \mathrm{~mm}$, and for a range of crack lengths between $a=1 \mathrm{~mm}$ to $a=25 \mathrm{~mm}$, for both values for $h$. The tests were conducted at a displacement rate of $1 \mathrm{~mm} \mathrm{~s}^{-1}$ for the specimens with $h=5 \mathrm{~mm}$, and at a rate of $0.2 \mathrm{~mm} \mathrm{~s}^{-1}$ for the specimens with $h=1 \mathrm{~mm}$, to ensure a constant value of strain rate within the cellulose acetate tape (remote from the crack tip). At least three tests per joint geometry were conducted.

Additional measurements were performed to characterize the mechanical properties of the cellulose acetate tape, at nominal strain rates ranging from $10^{-3} \mathrm{~s}^{-1}$ to $10^{-1} \mathrm{~s}^{-1}$. A flat dogbone geometry, as shown in Figure 11a, with the longitudinal direction of the tape aligned along the

\footnotetext{
${ }^{4}$ Scotch Magic 810 tape from 3M (Maplewood, US). The adhesive thickness is negligible compared to that of the cellulose acetate.

${ }^{5}$ AT500 tape from Advance Tapes International Ltd (Thurmaston, UK). The adhesive layer thickness is negligible to that of the aluminium alloy.
} 
loading direction, was used to determine the nominal stress-strain curve. At least three uniaxial tensile tests were conducted for each strain rate. The nominal stress-strain curve was also measured in uniaxial tension at a nominal strain rate of $10^{-1} \mathrm{~s}^{-1}$ using the strip specimen shown in Figure 11b, for which the longitudinal direction of the tape was orthogonal to the loading direction. The nominal strain in the uniaxial tensile tests was measured using a laser extensometer over a gauge length of $10 \mathrm{~mm}$.

The critical mode-I (plane-stress) stress-intensity factor $K_{\mathrm{c}}$ of the cellulose acetate tape was measured using a double edge-notched tension (DENT) specimen ${ }^{6}$. The T-L DENT specimen (defined in Figure 11c) contained an initial pre-crack aligned with the longitudinal direction of the tape, whereas the L-T DENT specimen (defined in Figure 11d) contained an initial pre-crack aligned with the transverse direction of the tape. The pre-cracks were cut with a sharp razor blade, and the plane-stress, mode I fracture toughness $K_{\mathrm{c}}$ was calculated by making use of the relation

$$
K_{\mathrm{c}}=F \sigma_{\mathrm{f}}^{\infty} \sqrt{\pi a},
$$

where $\sigma_{\mathrm{f}}^{\infty}$ is the tensile failure strength of the specimen corresponding to the peak load, and the finite width-correction factor $F(a / W)$ is (Benthem and Koiter, 1972; Tada et al., 2000)

$$
F=\left[1.122-0.561 \frac{a}{W}-0.205\left(\frac{a}{W}\right)^{2}+0.471\left(\frac{a}{W}\right)^{3}-0.19\left(\frac{a}{W}\right)^{4}\right]\left(1-\frac{a}{W}\right)^{-1 / 2} \text {. }
$$

The length $a$ of the pre-crack ranged from $a=1 \mathrm{~mm}$ to $a=6 \mathrm{~mm}$ for both the T-L and the L-T DENT specimens. Two tests were conducted for each value of $a$ and for each geometry, using a cross-head speed of $1 \mathrm{~mm} \mathrm{~s}^{-1}$.

Write $\dot{P}$ and $\dot{K}^{\infty}$ as the time-rate of change of load and of the remote stress intensity factor, respectively. Then, $\dot{K}^{\infty}$ is given by

$$
\dot{K}^{\infty}=\dot{P} \frac{(\pi a)^{\frac{1}{2}}}{w t}
$$

\footnotetext{
${ }^{6}$ It is shown in the following section that the plastic zone size for a long crack in the cellulose acetate tape is close to $1 \mathrm{~mm}$. This value exceeds the tape thickness of $40 \mu \mathrm{m}$, thereby justifying the plane stress analysis assumption.
} 
where $w$ and $t$ refer to the nominal width and thickness, respectively, of the DENT specimens. The dependence of $K_{\mathrm{c}}$ upon $\dot{K}^{\infty}$ for the T-L DENT specimen geometry of Figure 11c was investigated by selected values of the cross-head speed from $0.3 \mathrm{~mm} \mathrm{~s}^{-1}$ to $6 \mathrm{~mm} \mathrm{~s}^{-1}$ for an initial crack length $a=2 \mathrm{~mm}$.

\subsection{Material characteristics and sandwich specimen strength}

Tensile and fracture toughness responses of the cellulose acetate tape

The nominal stress $\sigma_{\mathrm{n}}$ is plotted as a function of nominal strain $\varepsilon_{\mathrm{n}}$ in Figure 12 for both tensile geometries. A small degree of anisotropy is evident. The measured values ${ }^{7,8}$ of Young's modulus $E$ and yield strength $\sigma_{\mathrm{y}}$ for each strain rate are reported in Table 1 . The measured load versus cross-head displacement curves of the DENT specimens were linear in nature (not shown), and the maximum load corresponded to brittle fracture with unstable crack propagation from the tip of the pre-crack. The measured plane stress fracture toughness $K_{\mathrm{c}}$ of the cellulose acetate tape is plotted as a function of initial crack length $a$ / $W$ in Figure 13a. The effect of loading rate upon $K_{\mathrm{c}}$ is reported in Figure 13b. Within the range explored, the value of $K_{\mathrm{c}}$ is independent of crack length, crack orientation and loading rate. Based upon a total of 34 individual measurements, $K_{\mathrm{c}}=3.0 \pm 0.4 \mathrm{MPa} \sqrt{\mathrm{m}}$ (where the uncertainty corresponds to a 95\% confidence level of two standard deviations).

Tensile strength of the sandwich layer

The measured failure strength $\sigma_{\mathrm{f}}^{\infty} / \hat{\sigma}$ of the centre-cracked cellulose acetate-aluminium sandwich layer is reported in Figure 14 as a function of crack length $a / h$ for $h / W=0.1$ and $h / W$ $=0.02$. For this plot, we have assumed that $\hat{\sigma}$ equals $54 \mathrm{MPa}$, corresponding to the average tensile yield strength of the strip specimen (see Figure 12) at a strain rate of $10^{-1} \mathrm{~s}^{-1}$.

In order to include predictions according to (35) in Figure 14, it is first necessary to deduce the value of $l_{\mathrm{s}}$. This, and other pertinent material properties, were estimated as follows. The

\footnotetext{
${ }^{7}$ The Young's modulus is based on the slope of the initial linear part of the measured nominal stress versus nominal strain curves.

${ }^{8}$ The yield strength corresponds to the value of the peak load subsequent to the initial linear, elastic regime of the measured load versus cross-head displacement curve.
} 
elastic constants, $\bar{E}$ and $\bar{v}$ are simply taken as their plane-stress values of $E$ and $v$. At the appropriate strain rate, $E_{2}$ equals $6.4 \mathrm{GPa}$ and $v_{2}$ is taken to be 0.38 (Tsou et al., 1995). Upon assuming $K_{\mathrm{c}}=\left(E_{2} \Gamma\right)^{1 / 2}=3.0 \pm 0.4 \mathrm{MPa} \sqrt{\mathrm{m}}$, the material length scale for the cellulose acetate material is $l_{\mathrm{S}}=0.98 \pm 0.28 \mathrm{~mm}$ via (6). The modulus of aluminum is $E_{1}=70 \mathrm{GPa}$ and $v_{1}$ equals 0.33 (Callister, 2007), implying a modulus mismatch of $E_{2} / E_{1}=0.09$.

The predictions (35) are shown in Figure 14 for three assumed values of $l_{\mathrm{s}}$ as follows. The solid line and dotted lines for $h / W=0.02$ are for the mean value $l_{\mathrm{S}}=0.98 \mathrm{~mm}$ and the upper and lower limits ( $l_{\mathrm{s}}$ equals $1.26 \mathrm{~mm}$ and $0.70 \mathrm{~mm}$, respectively). Note that the mean value of $l_{S} / h$ equals 1 for this choice of $h / W=0.02$, and this implies that regime $D$ is active for $a / h<3$ while regime $C$ is active for $a / h>3$, recall Figure 9. The measured strengths broadly support this: the strength drops with increasing $a / h$ in the regime $C$ of toughness control. The strength ratio $\sigma_{\mathrm{f}}^{\infty} / \hat{\sigma}$ is close to 0.9 at $a / h<0.3$, which is within $10 \%$ of the plastic collapse load, $\sigma_{\mathrm{f}}^{\infty} / \hat{\sigma}=1$. The source of this minor discrepancy is unclear; the analysis neglects the strain rate sensitivity of $\hat{\sigma}$ and assumes perfect bonding between the cellulose acetate strip and the substrates. A detailed investigation into the sources of the discrepancy is beyond the scope of the present paper.

Now consider the case $h / W=0.1$. Then, the thick dashed assumes a mean value $l_{\mathrm{S}}=0.98$ $\mathrm{mm}$, whereas the dotted lines assume upper and lower limiting values of $l_{\mathrm{S}}$ equal to $1.26 \mathrm{~mm}$ and $0.70 \mathrm{~mm}$, respectively. Note that the mean value of $l_{\mathrm{S}} / h$ equals 0.2 for this choice of $h / W$ $=0.1$. Examination of the maps of Figures 7 and 9 reveals that regime $\mathrm{C}$ (toughness-control) dominates over strength control, regime D, and the measurements support this: the strength drops with increasing $a / h$ as predicted by (35), to within scatter. In summary, the analytical model, with the attendant fracture maps of Figures 7 and 9, is supported by the series of experiments, confirming the existing of the competing failure regimes.

\section{Concluding remarks}

Our study highlights a number of fundamental features of a cracked, elastic sandwich layer that have received minor attention in the literature to date. Most notably, a large region of almost constant tensile stress exists ahead of a crack tip, when the sandwich layer has a much 
lower Young's modulus than that of the substrate. Consequently, the existence of an outer K field for the cracked sandwich specimen may require a large specimen, or engineering structure. Comprehensive maps are presented to show regimes of failure mechanism as a function of elastic mismatch and material non-linearity (in deformation and fracture response), as modelled by a tensile cohesive zone at the crack tip.

The tensile strength of an adhesive joint, comprising an adhesive layer made from a linear, elastic material of Youngs modulus $E_{2}$ and two substrates made from a linear, elastic material of modulus $E_{1}$, is predicted as a function of crack length and material mismatch ratio, $E_{2} / E_{1}$ . Consider first the case of an elastic-brittle response for a Griffith crack. The relation between the normalized tensile strength and normalized crack length switches from an adhesivegoverned regime for the case where the crack is much smaller than the adhesive layer height to a substrate-governed regime for the case where the crack is much longer than the adhesive layer height. The normalized strength of the joint is independent of normalized crack length for intermediate crack lengths up to a value on the order of $h E_{1} / E_{2}$. The role of crack tip plasticity on the normalized strength versus normalized crack length trend is explored by the use of a strip yield model and design diagrams are constructed. The developed analytical theory is verified by finite element calculations. Further, the observed dependence of tensile strength of a sandwich layer upon crack length is validated by a series of tests on a centre-cracked cellulose acetate tape sandwiched between two aluminium alloy substrates.

\section{Acknowledgements}

Financial support from the Engineering and Physical Sciences Research Council (UK) award 1611305, the ERC MULTILAT grant 669764, and SABIC are gratefully acknowledged.

Table 1 Measured Young's modulus $E$ and yield strength $\sigma_{\mathrm{y}}$ of the cellulose acetate tape. The uncertainty corresponds to a 95\% confidence level of two standard deviations. The dogbone geometry is sketched in Figure 11a, and the strip geometry is sketched in Figure 11b. 


\begin{tabular}{|c|c|c|c|}
\hline$\dot{\varepsilon}_{\mathrm{n}}\left(\mathrm{s}^{-1}\right)$ & $E(\mathrm{GPa})$ & $\sigma_{\mathrm{y}}(\mathrm{MPa})$ & Geometry \\
\hline $10^{-3}$ & $1.7 \pm 0.6$ & $36.1 \pm 2.8$ & Dogbone \\
\hline $10^{-2}$ & $3.2 \pm 0.9$ & $45.3 \pm 3$ & Dogbone \\
\hline $10^{-1}$ & $7.1 \pm 0.8$ & $56.1 \pm 4.4$ & Dogbone \\
\hline $10^{-1}$ & $6.4 \pm 1.0$ & $53.9 \pm 4.8$ & Strip \\
\hline
\end{tabular}




\section{List of figure captions}

Figure 1 Cross-sectional view of a sandwich joint consisting of an adhesive layer of thickness $2 h$, with a through-thickness crack of length $2 a$, and loaded by a remote stress $\sigma^{\infty}$. The traction-separation law for the cohesive zone in the adhesive is shown on the right, and results in a cohesive zone of length $c$ at the crack tip.

Figure 2 A linear-elastic adhesive layer (material 2) of height $2 h$ is sandwiched between two linear-elastic substrates (material 1). A semi-infinite crack in the adhesive layer exists on the mid-plane of the adhesive layer, parallel to the interfaces. The joint is subjected to a remote mode I loading of magnitude, $K^{\infty}$.

Figure 3 The tensile stress distribution directly ahead of a semi-infinite crack in a sandwich layer. (a) The tensile stress is normalized by $K^{\infty}$ and the modulus mismatch ratio is $\bar{E}_{2} / \bar{E}_{1}=10^{-2}$; (b) The tensile stress is normalized by $K^{\text {tip }}$.

Figure 4 Superposition procedure to calculate the $K$-calibration for a Griffith crack in an elastic layer.

Figure 5 Strength $\bar{\sigma}$ versus normalized crack length $a / h$ for an elastic-brittle Griffith crack, (a) for $h / W=0$ and (b) for $\bar{E}_{1} / \bar{E}_{2}=10^{-2}$. The dotted lines are the asymptote (12), while the solid lines are (23) in regime $\mathrm{C}$ and (10) in regime $\mathrm{B}$.

Figure 6 Superposition procedure to calculate the macroscopic tensile strength of a centre-cracked sandwich layer, for finite values of $l_{\mathrm{S}}$, and $a$ is comparable to, or larger than $h$.

Figure 7 Failure mechanism map for centre-cracked sandwich specimen for $h / W=0$ and the 2 choices $\bar{E}_{2} / \bar{E}_{1}=10^{-2}$ (solid line) and $\bar{E}_{2} / \bar{E}_{1}=0$ (dashed line). The contours of strength are given by (36) in regimes $C$ and $D$, and by (27) in regimes $A$ and $B$.

Figure 8 Strength predictions for $h / W=0$, and $\bar{E}_{2} / \bar{E}_{1}=10^{-2}$ (solid line) or $\bar{E}_{2} / \bar{E}_{1}=0$ (dashed line) (a) $\sigma_{\mathrm{f}}^{\infty} / \hat{\sigma}$ versus $a / h$; (b) $\bar{\sigma}$ versus $a / h$. In both figures, predictions are given by (27) for $l_{\mathrm{S}} / h \leq 0.1$ and $a / h<0.3$. Otherwise, (36) is used. 
Figure 9 Boundary between regimes $C$ and $D$ in a map of $l_{\mathrm{S}} / h$ versus $\bar{E}_{2} / \bar{E}_{1}$, for selected values of $a / h$, for $h / W=0$. The two data points denote the experimental parameters that are measured for cellulose acetate- aluminium alloy in the experimental study.

Figure 10 Sandwich specimen made from cellulose acetate tape containing a centre-crack of length $2 a$ adhered to a tape of an aluminium alloy. $L=35 \mathrm{~mm}$ and $W=50 \mathrm{~mm}$. The double arrow gives the longitudinal direction of the tape.

Figure 11 Materials tests on cellulose acetate tape. (a) Dogbone geometry with the tensile load applied along the longitudinal direction of the tape ( $W=24 \mathrm{~mm}, w=6.4 \mathrm{~mm}, h=40$ $\mathrm{mm}, L=50 \mathrm{~mm}, R=15.6 \mathrm{~mm}$ ). (b) A strip geometry with the tensile load applied along the transverse direction of the tape ( $w=10 \mathrm{~mm}, h=14 \mathrm{~mm})$. (c) A T-L DENT specimen with $w$ $=24 \mathrm{~mm}, h=10 \mathrm{~mm}$, and (d) a L-T DENT specimen with $w=24 \mathrm{~mm}$.

Figure 12 Nominal stress, $\sigma_{\mathrm{n}}$, plotted as a function of nominal strain, $\varepsilon_{\mathrm{n}}$, for the cellulose acetate tape in uniaxial tension at 3 nominal strain rates using the dogbone specimen of Figure 11a, and at a nominal strain rate of $10^{-1} \mathrm{~s}^{-1}$ for the strip (S) specimen of Figure 11b, as indicated by "S" on this plot. Crosses at the end of the curve denote failure of the specimen.

Figure 13 Plane-stress, fracture-toughness measurements for the cellulose acetate tape: (a) $K_{\text {c }}$ versus $a / W$, for the geometries shown in Figure 11 c and $11 \mathrm{~d}$; (b) $K_{\text {c }}$ versus $\dot{K}$. Crack length $a$ is measured to an accuracy of $0.2 \mathrm{~mm}$. The mean and range of fracture toughness values are included in each figure as a solid line and dotted lines, respectively.

Figure 14 Measured and predicted strength $\sigma_{\mathrm{f}}^{\infty} / \hat{\sigma}$ of sandwich specimens versus $a / h$ for $h / W=0.1$ and $h / W=0.02$. The values of $l_{\mathrm{S}} / h$ are 0.2 and 1 for $h / W$ equal to 0.1 and 0.02 , respectively. 


\section{Figures}

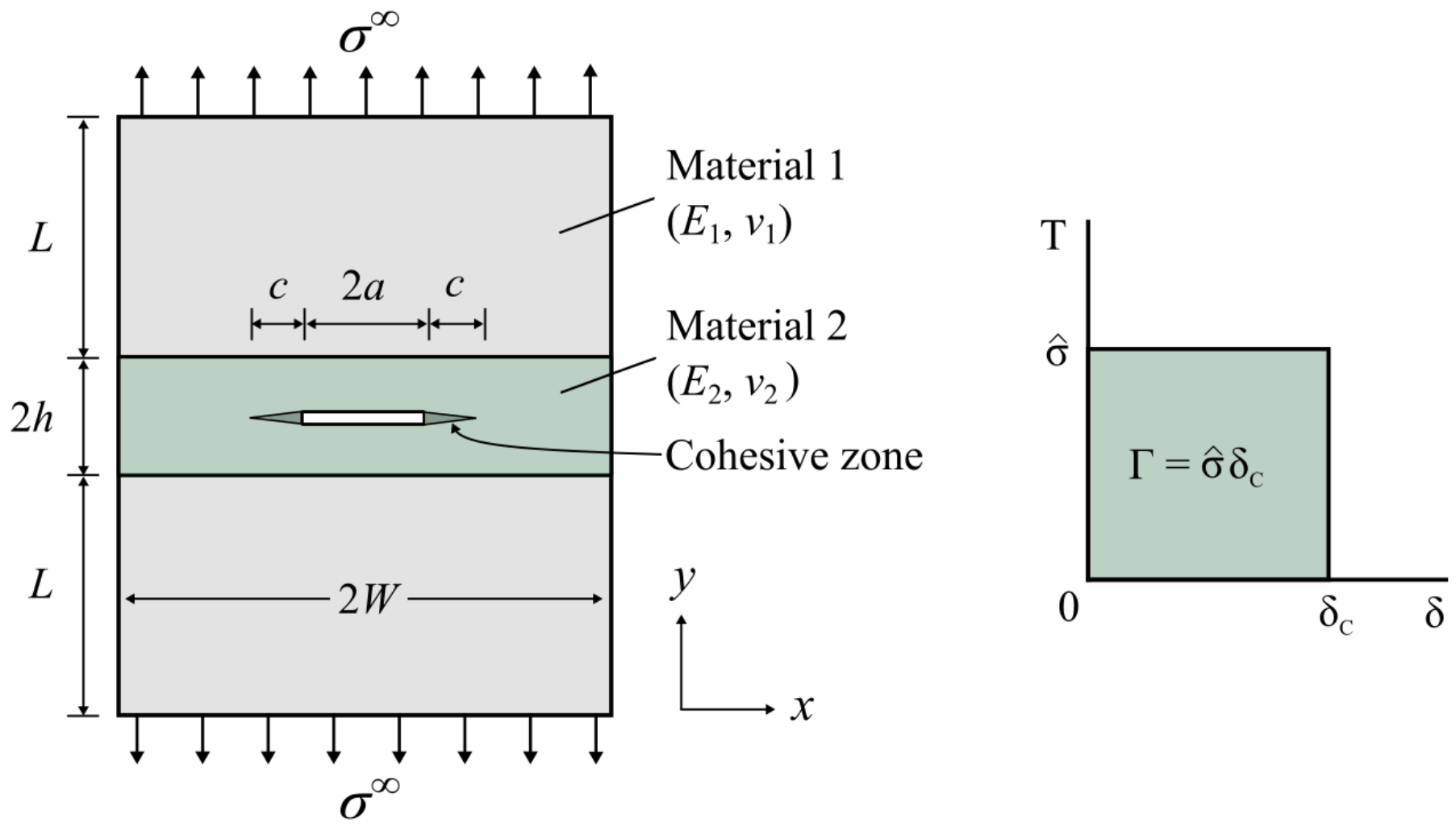

Figure 1 Cross-sectional view of a sandwich joint consisting of an adhesive layer of thickness $2 h$, with a through-thickness crack of length $2 a$, and loaded by a remote stress $\sigma^{\infty}$. The traction-separation law for the cohesive zone in the adhesive is shown on the right, and results in a cohesive zone of length $c$ at the crack tip. 


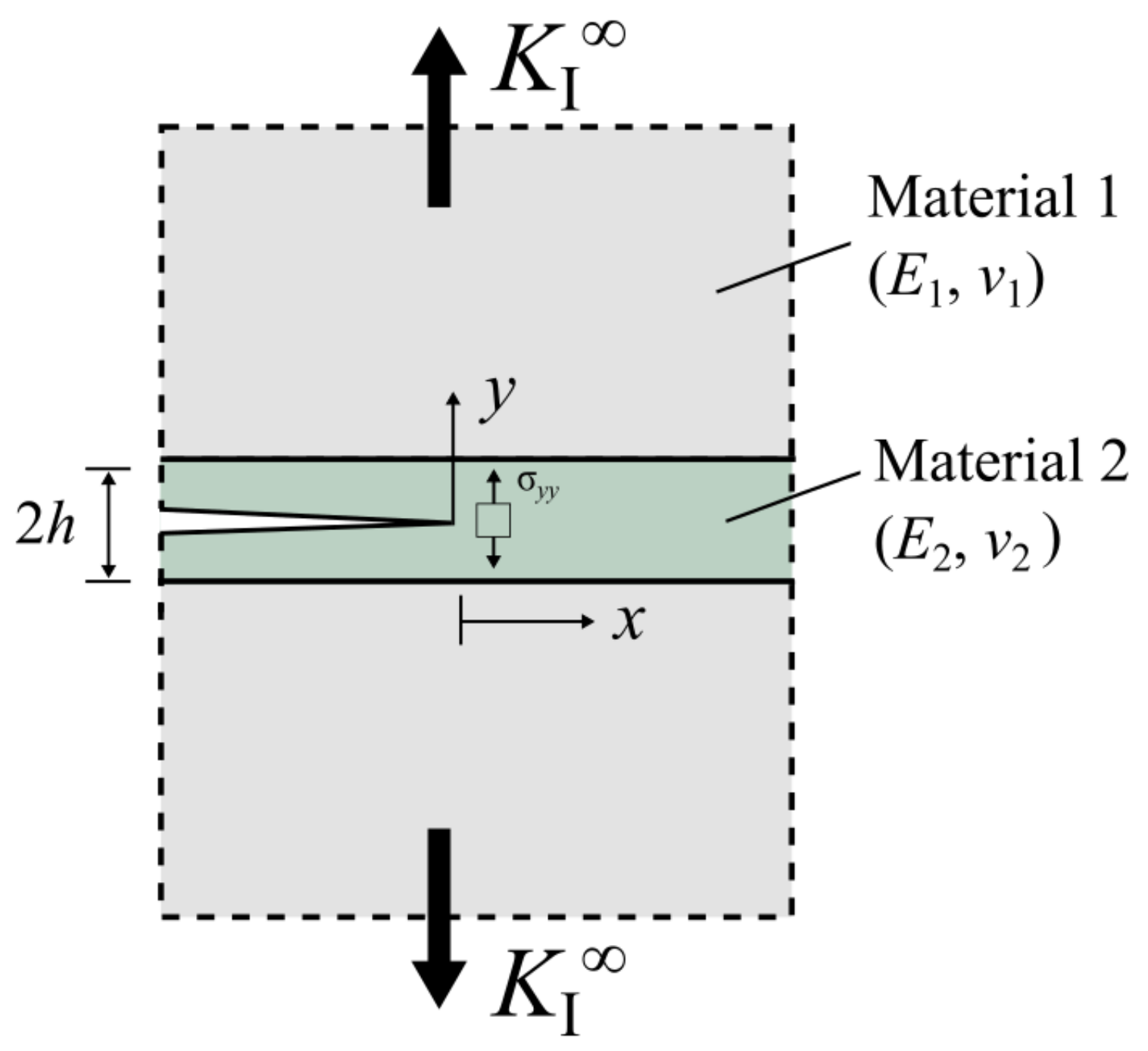

Figure 2 A linear-elastic adhesive layer (material 2) of height $2 h$ is sandwiched between two linear-elastic substrates (material 1). A semi-infinite crack in the adhesive layer exists on the mid-plane of the adhesive layer, parallel to the interfaces. The joint is subjected to a remote mode I loading of magnitude, $K^{\infty}$. 

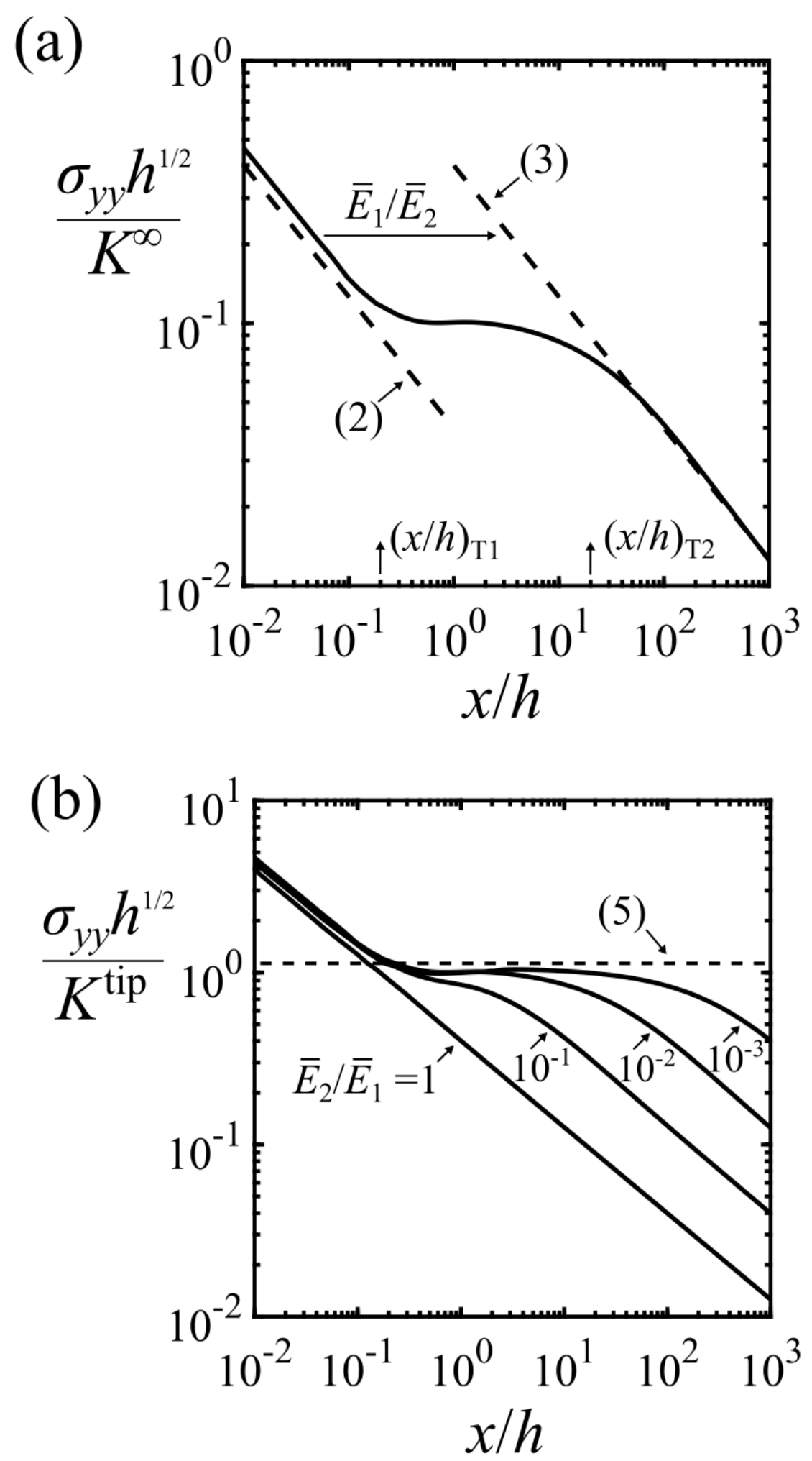

Figure 3 The tensile stress distribution directly ahead of a semi-infinite crack in a sandwich layer. (a) The tensile stress is normalized by $K^{\infty}$ and the modulus mismatch ratio is $\bar{E}_{2} / \bar{E}_{1}=10^{-2}$; (b) The tensile stress is normalized by $K^{\text {tip }}$. 


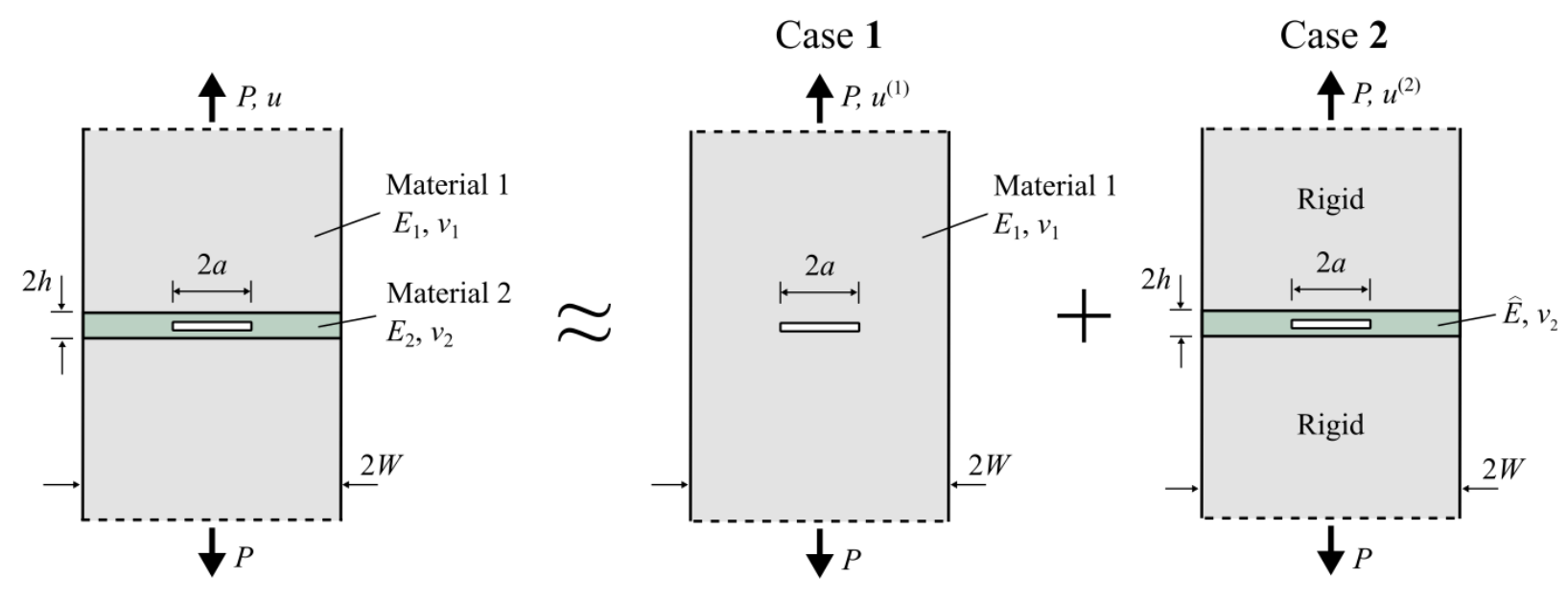

Figure 4 Superposition procedure to calculate the $K$-calibration for a Griffith crack in an elastic layer. 

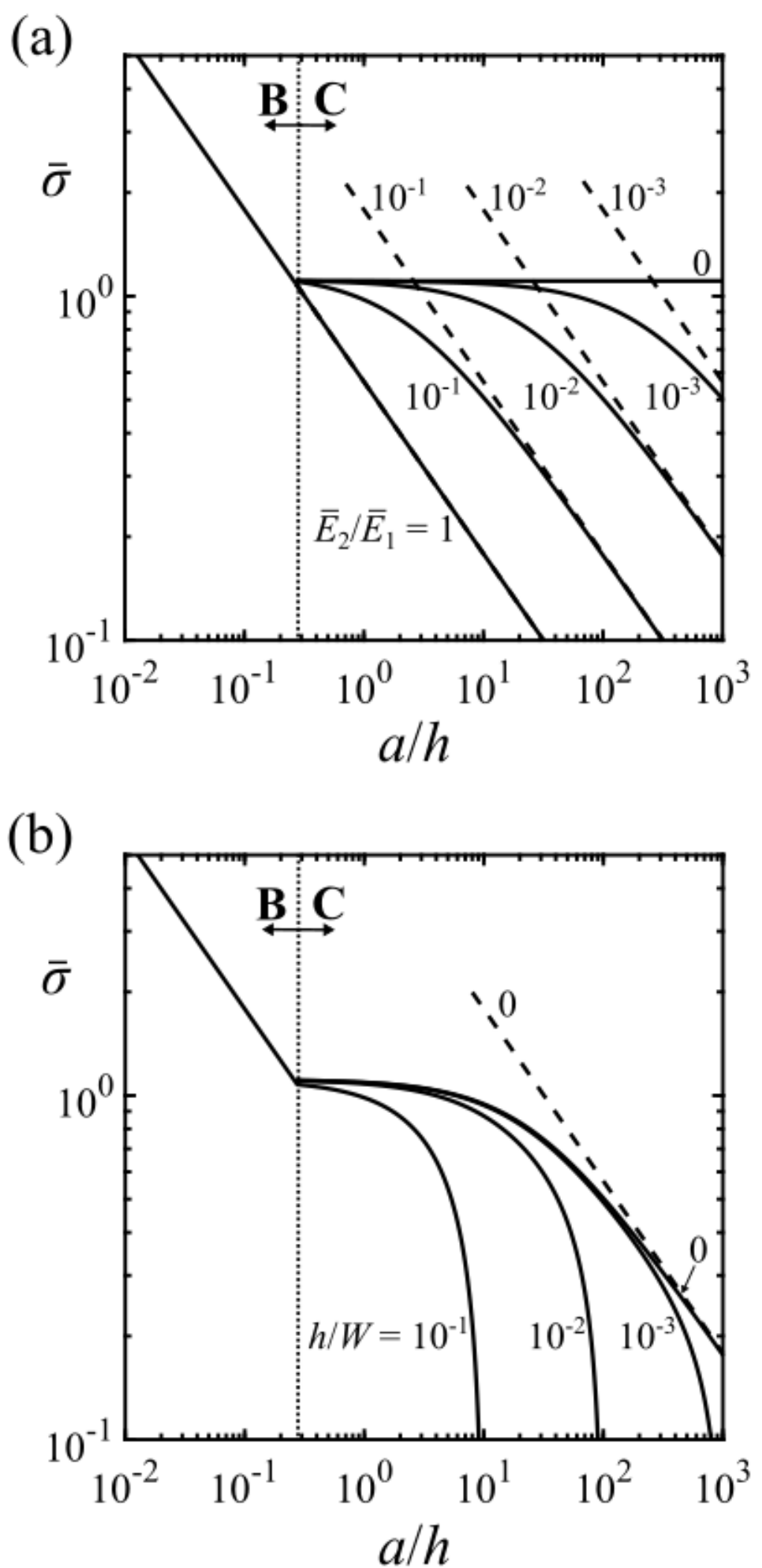

Figure 5 Strength $\bar{\sigma}$ versus normalized crack length $a / h$ for an elastic-brittle Griffith crack, (a) for $h / W=0$ and (b) for $\bar{E}_{1} / \bar{E}_{2}=10^{-2}$. The dotted lines are the asymptote (12), while the solid lines are (23) in regime $C$ and (10) in regime B. 


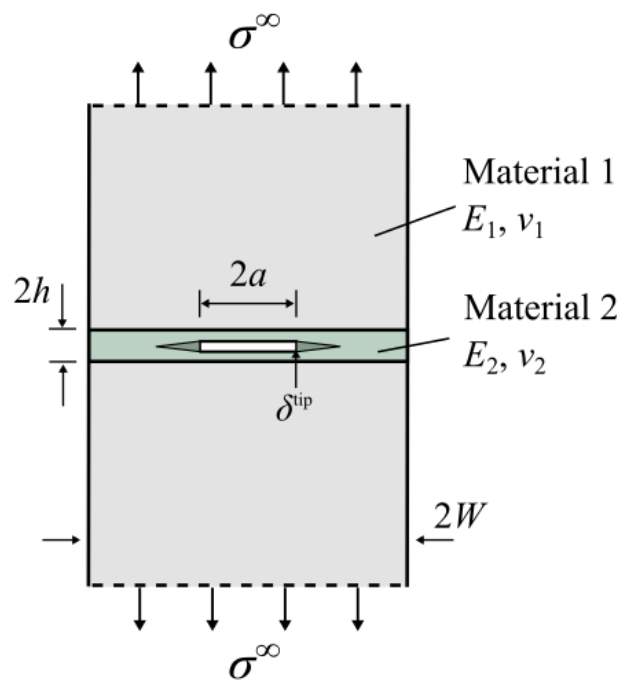

Case 1

Case 2

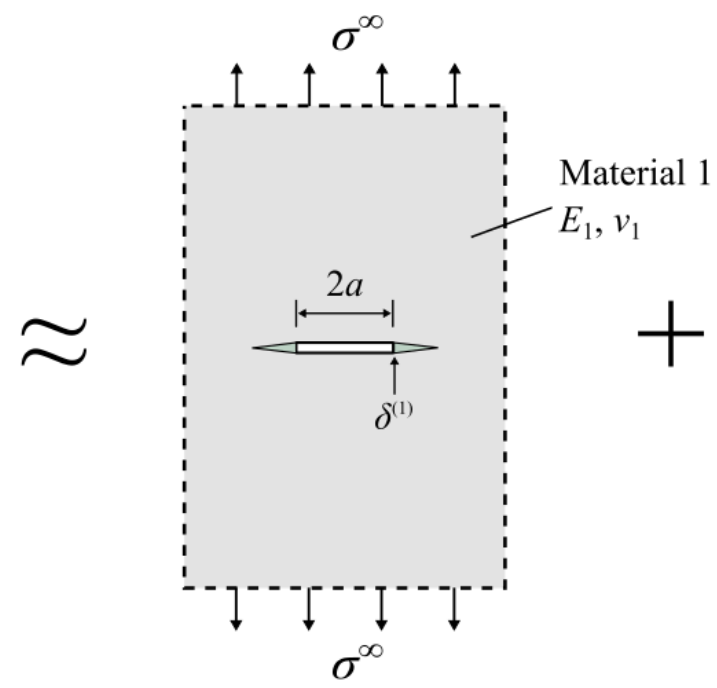

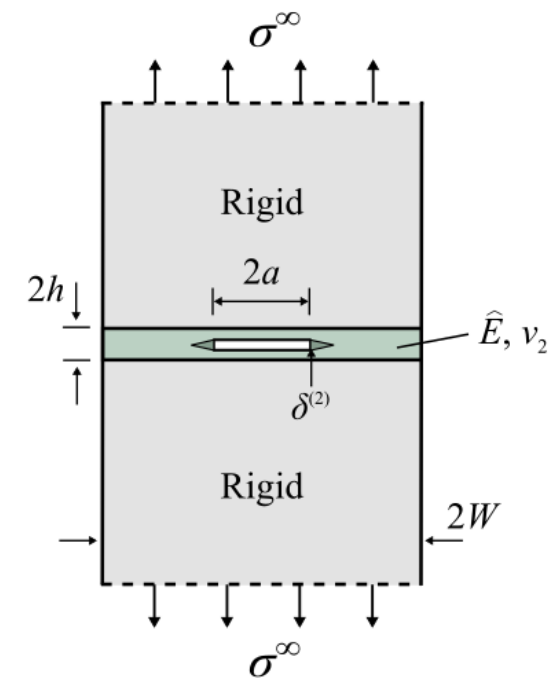

Figure 6 Superposition procedure to calculate the macroscopic tensile strength of a centre-cracked sandwich layer, for finite values of $l_{\mathrm{S}}$, and $a$ is comparable to, or larger than $h$. 

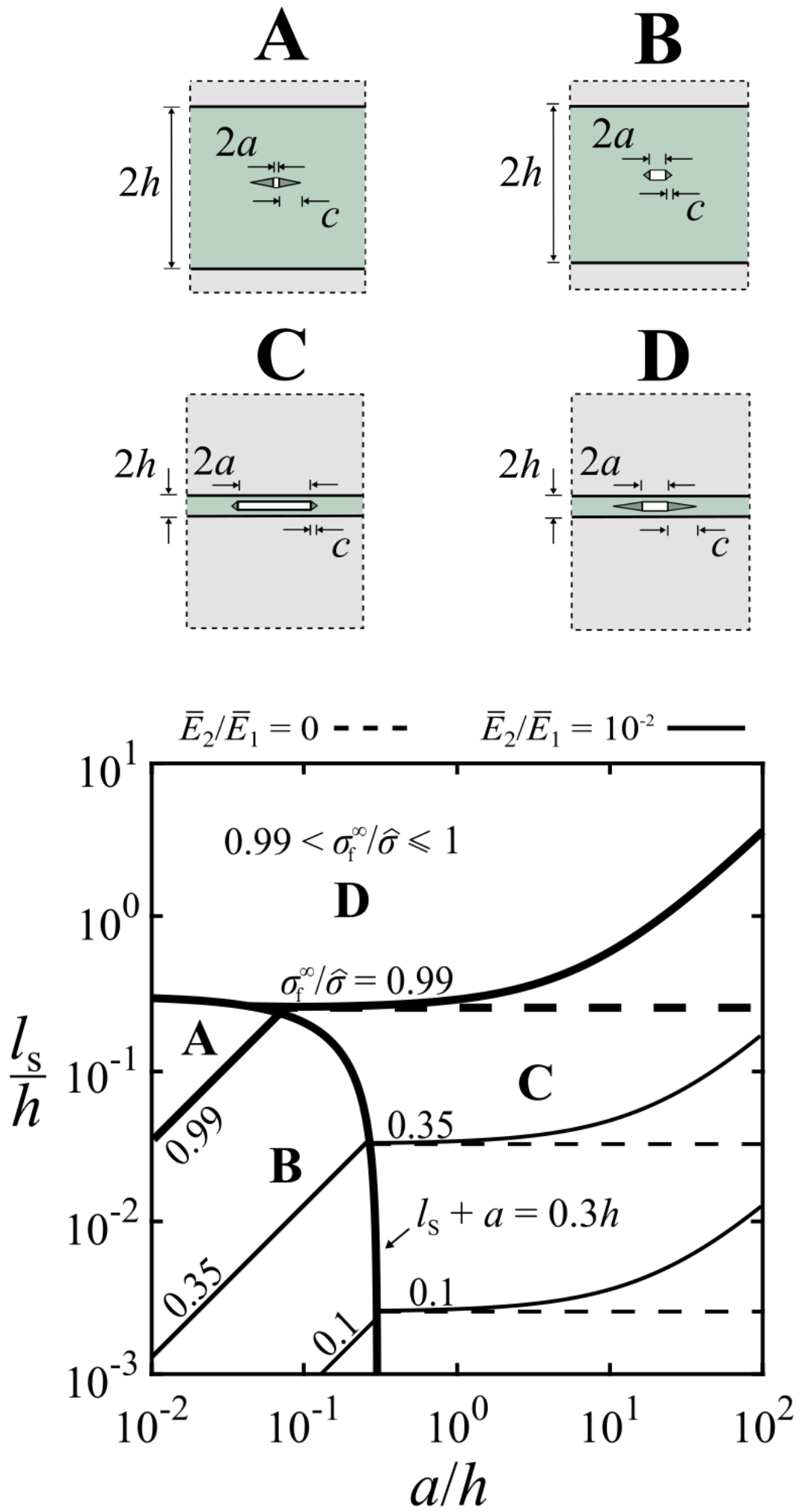

Figure 7 Failure mechanism map for centre-cracked sandwich specimen for $h / W=0$ and the 2 choices $\bar{E}_{2} / \bar{E}_{1}=10^{-2}$ (solid line) and $\bar{E}_{2} / \bar{E}_{1}=0$ (dashed line). The contours of strength are given by (36) in regimes $C$ and $D$, and by (27) in regimes $\mathrm{A}$ and $\mathrm{B}$. 


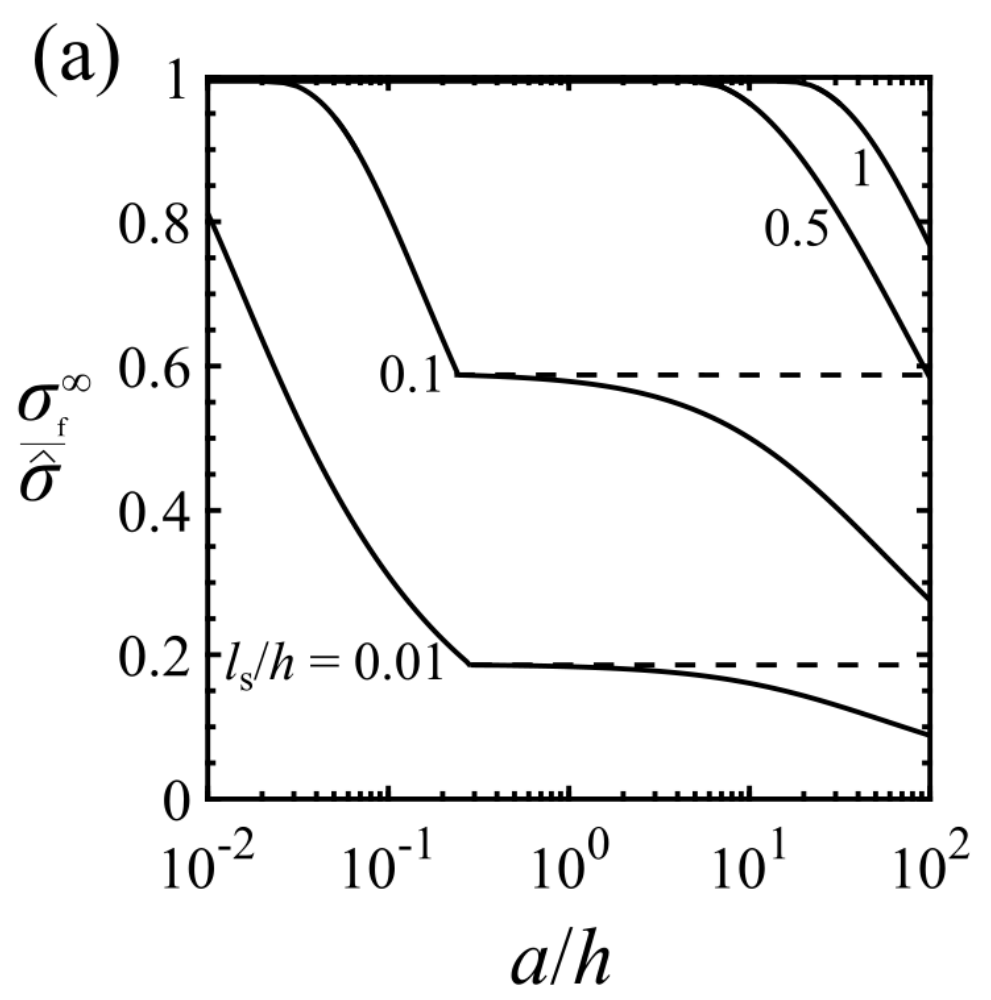

(b)

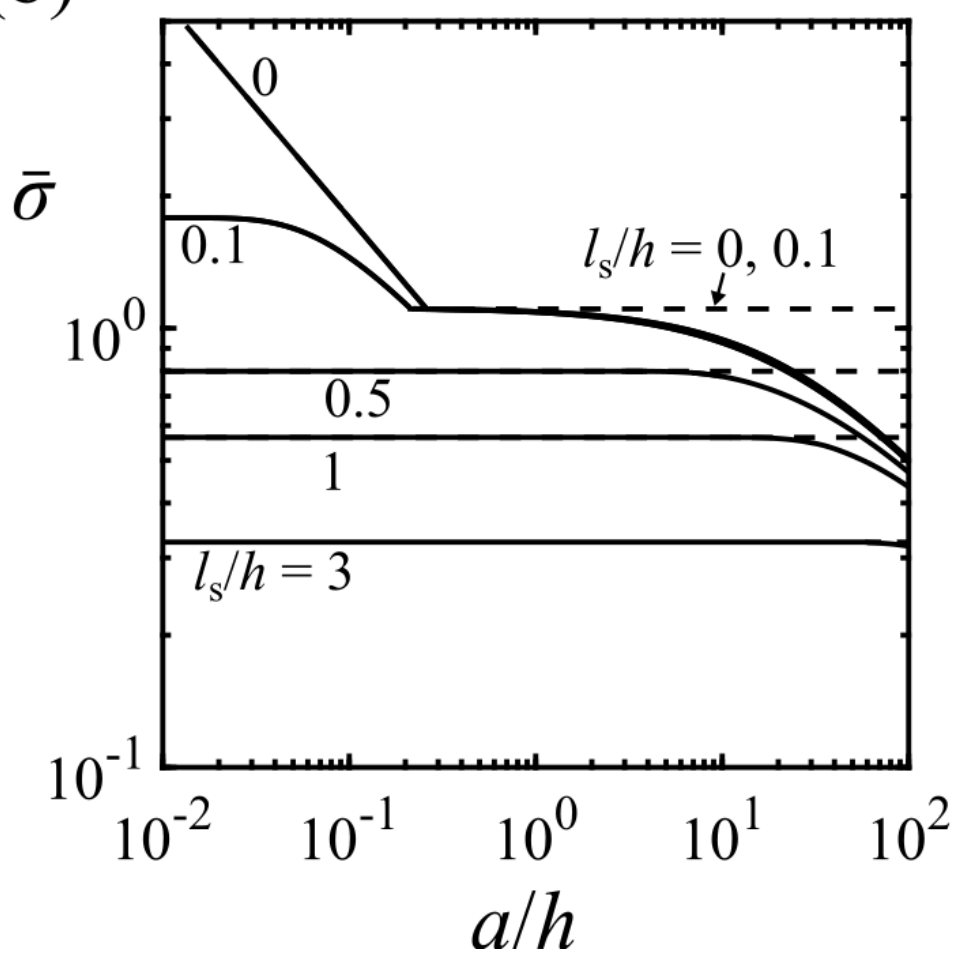

Figure 8 Strength predictions for $h / W=0$, and $\bar{E}_{2} / \bar{E}_{1}=10^{-2}$ (solid line) or $\bar{E}_{2} / \bar{E}_{1}=0$ (dashed line) (a) $\sigma_{\mathrm{f}}^{\infty} / \hat{\sigma}$ versus $a / h$; (b) $\bar{\sigma}$ versus $a / h$. In both figures, predictions are given by (27) for $l_{\mathrm{S}} / h \leq 0.1$ and $a / h<0.3$. Otherwise, (36) is used. 


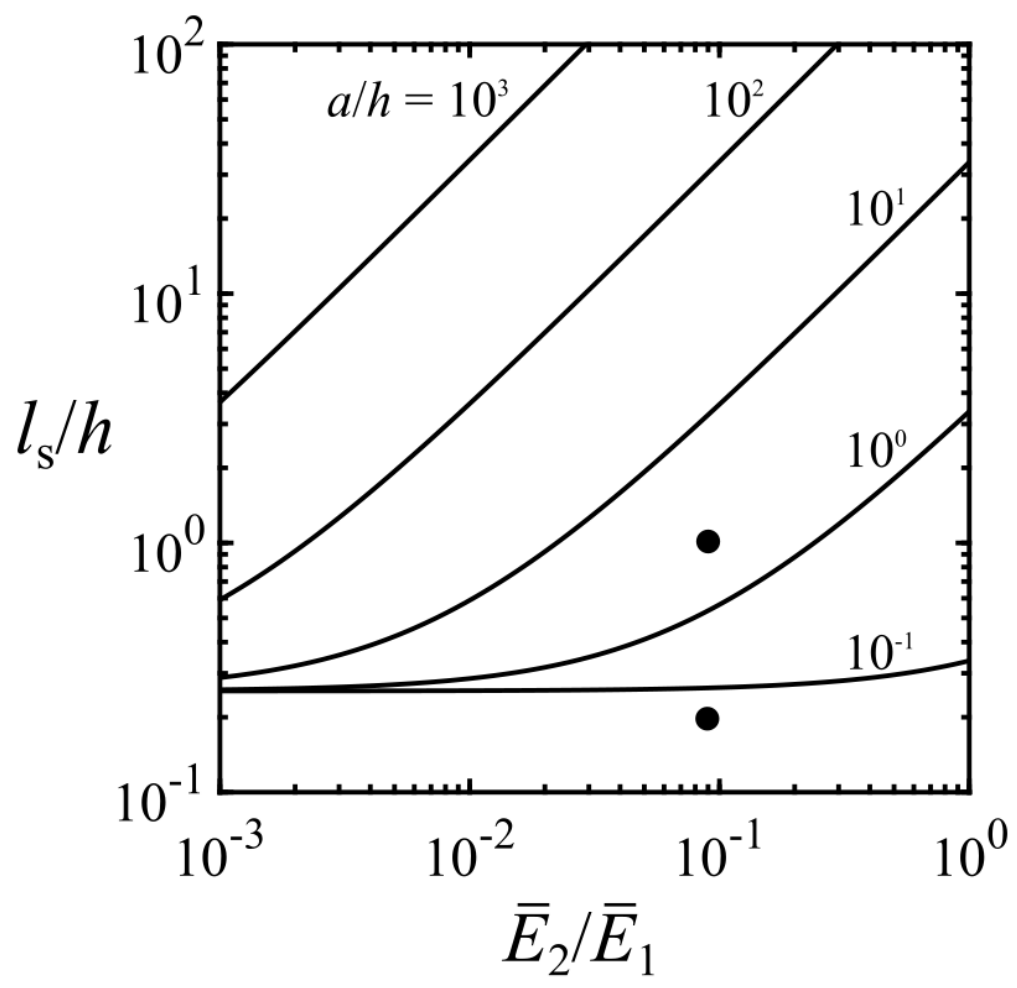

Figure 9 Boundary between regimes $C$ and $D$ in a map of $l_{\mathrm{S}} / h$ versus $\bar{E}_{2} / \bar{E}_{1}$, for selected values of $a / h$, for $h / W=0$. The two data points denote the experimental parameters that are measured for cellulose acetate- aluminium alloy in the experimental study. 


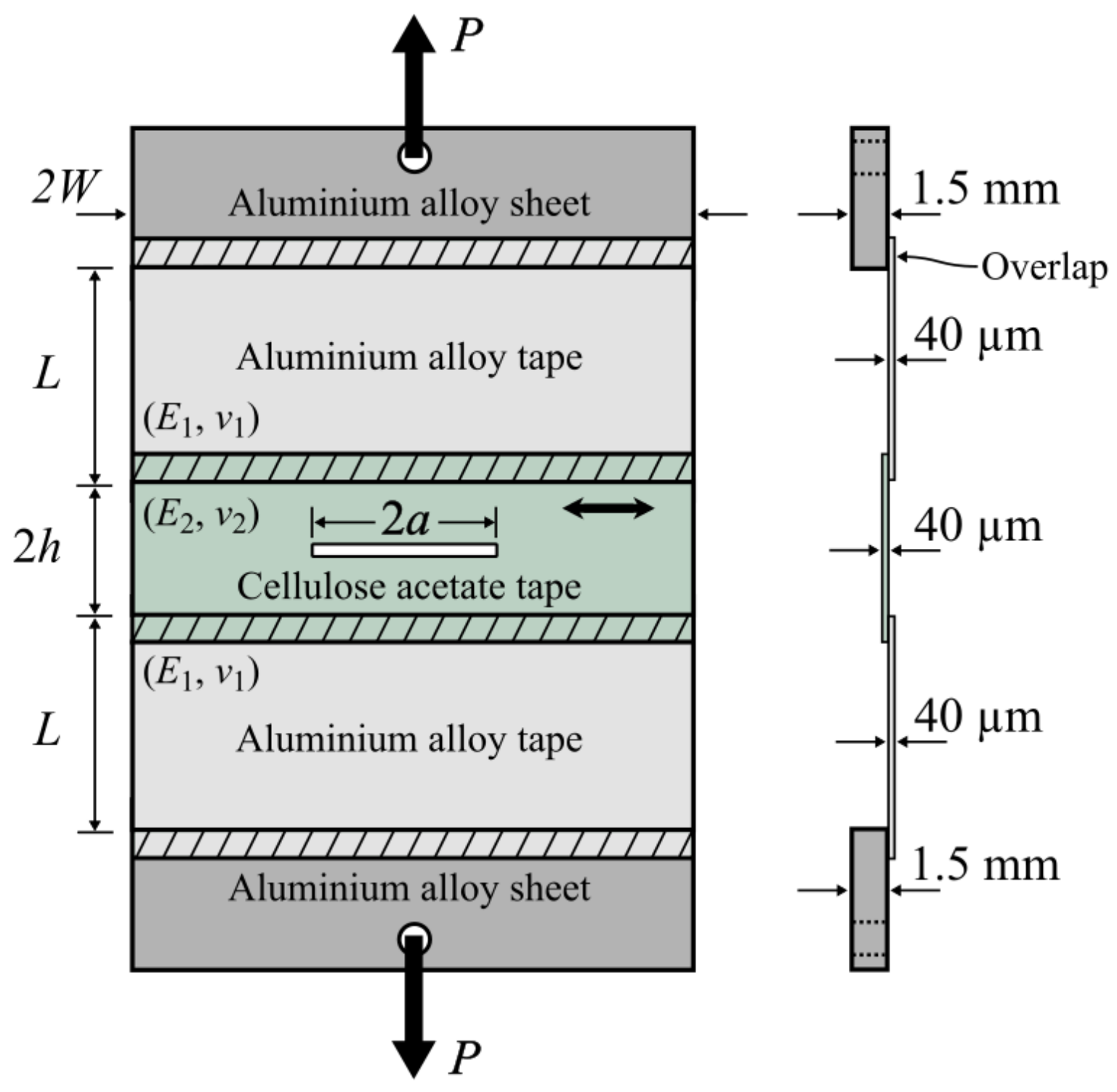

Figure 10 Sandwich specimen made from cellulose acetate tape containing a centre-crack of length $2 a$ adhered to a tape of an aluminium alloy. $L=35 \mathrm{~mm}$ and $W=50$ $\mathrm{mm}$. The double arrow gives the longitudinal direction of the tape. 
(a)

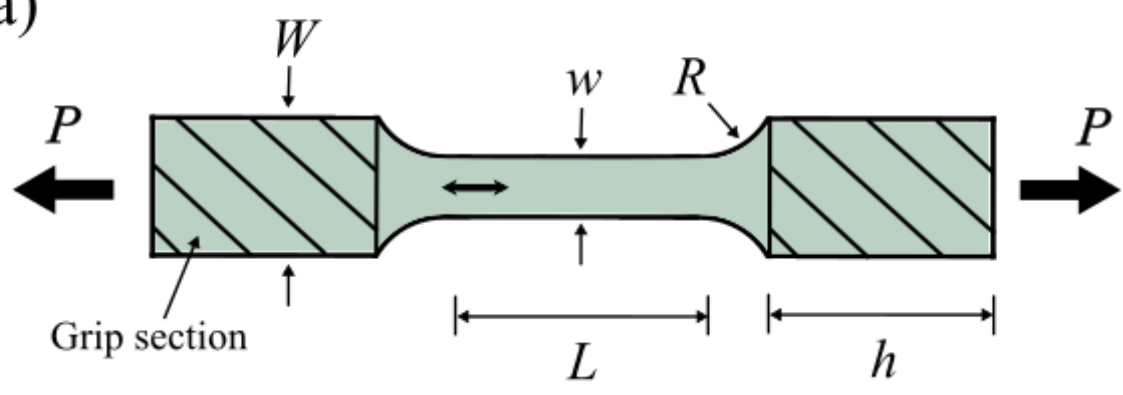

(b)

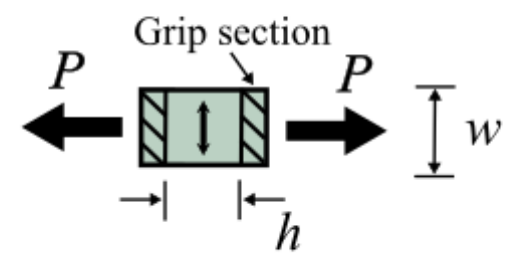

(d) T-L
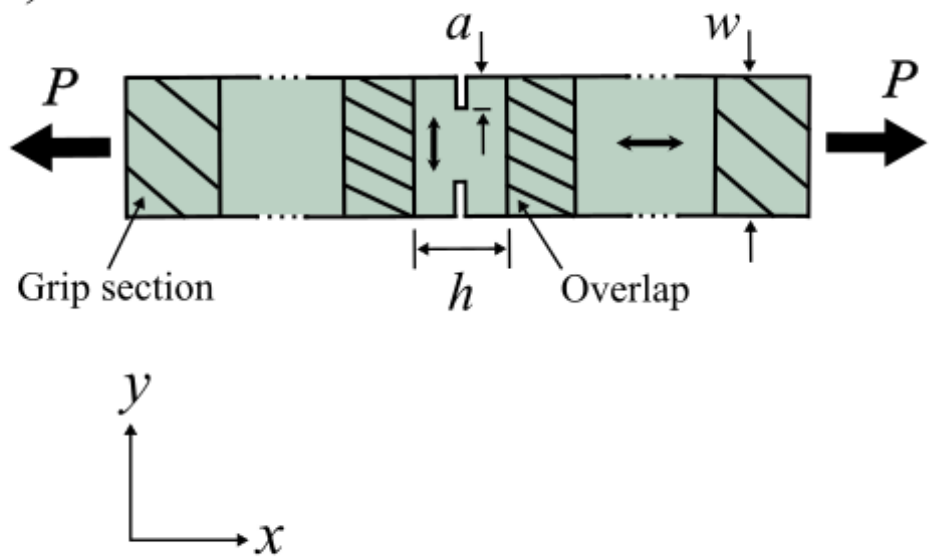

(d) L-T

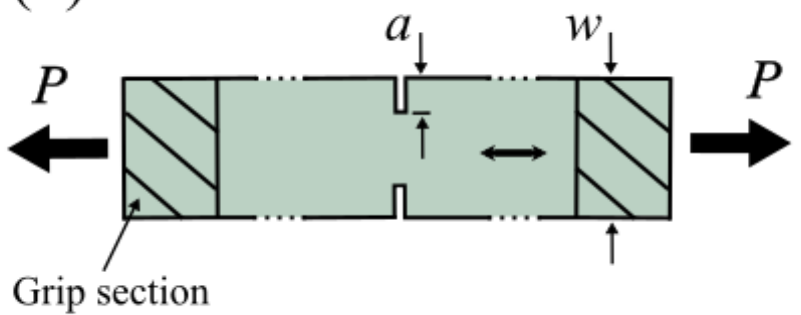

Grip section

\section{Arrow gives longitudinal direction of tape}

Figure 11 Materials tests on cellulose acetate tape. (a) Dogbone geometry with the tensile load applied along the longitudinal direction of the tape $(W=24 \mathrm{~mm}, w=6.4$ $\mathrm{mm}, h=40 \mathrm{~mm}, L=50 \mathrm{~mm}, R=15.6 \mathrm{~mm}$ ). (b) A strip geometry with the tensile load applied along the transverse direction of the tape $(w=10 \mathrm{~mm}, h=$ $14 \mathrm{~mm}$ ). (c) A T-L DENT specimen with $w=24 \mathrm{~mm}, h=10 \mathrm{~mm}$, and (d) a LT DENT specimen with $w=24 \mathrm{~mm}$. 


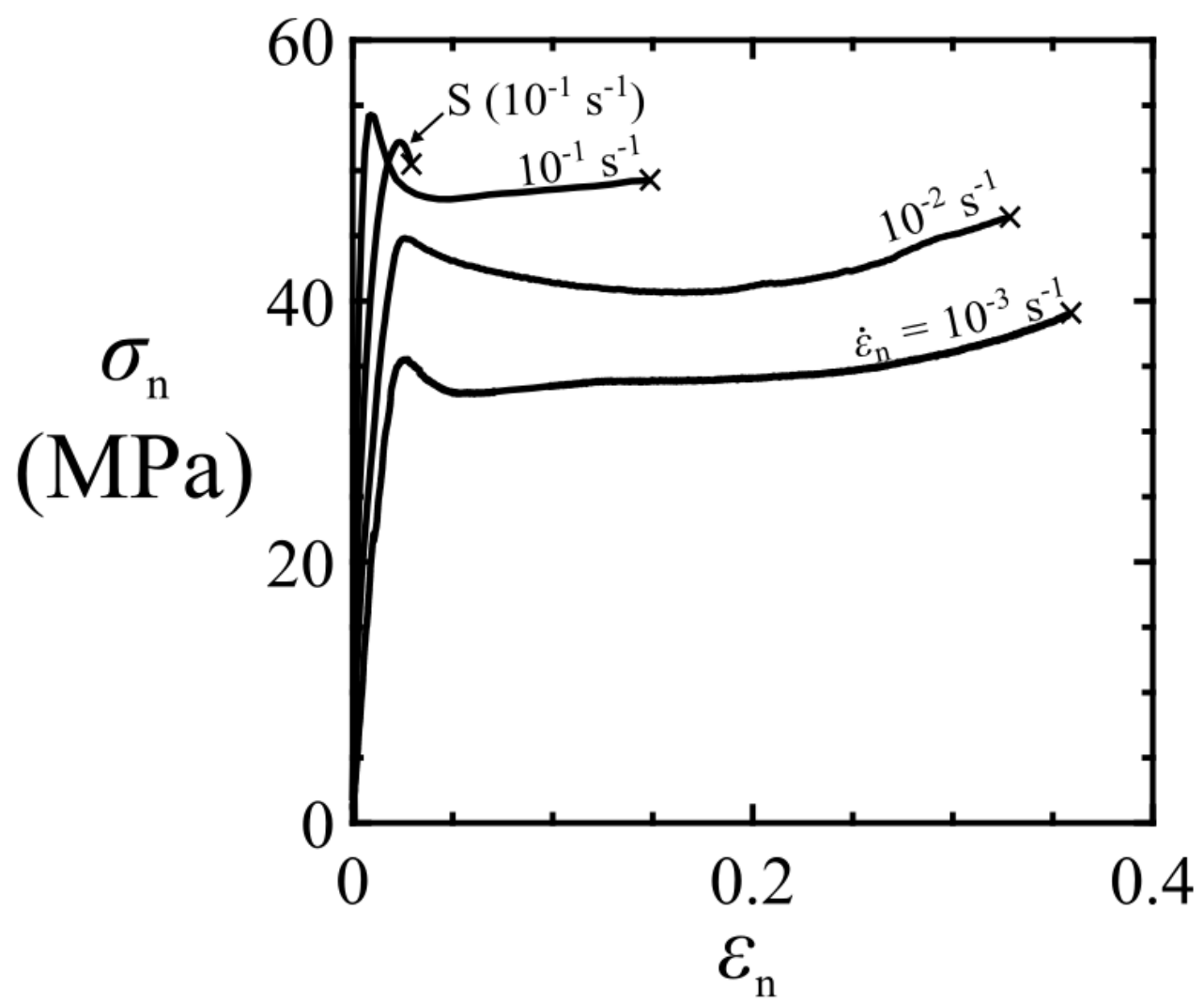

Figure 12 Nominal stress, $\sigma_{\mathrm{n}}$, plotted as a function of nominal strain, $\varepsilon_{\mathrm{n}}$, for the cellulose acetate tape in uniaxial tension at 3 nominal strain rates using the dogbone specimen of Figure 11a, and at a nominal strain rate of $10^{-1} \mathrm{~s}^{-1}$ for the strip (S) specimen of Figure 11b, as indicated by "S" on this plot. Crosses at the end of the curve denote failure of the specimen. 

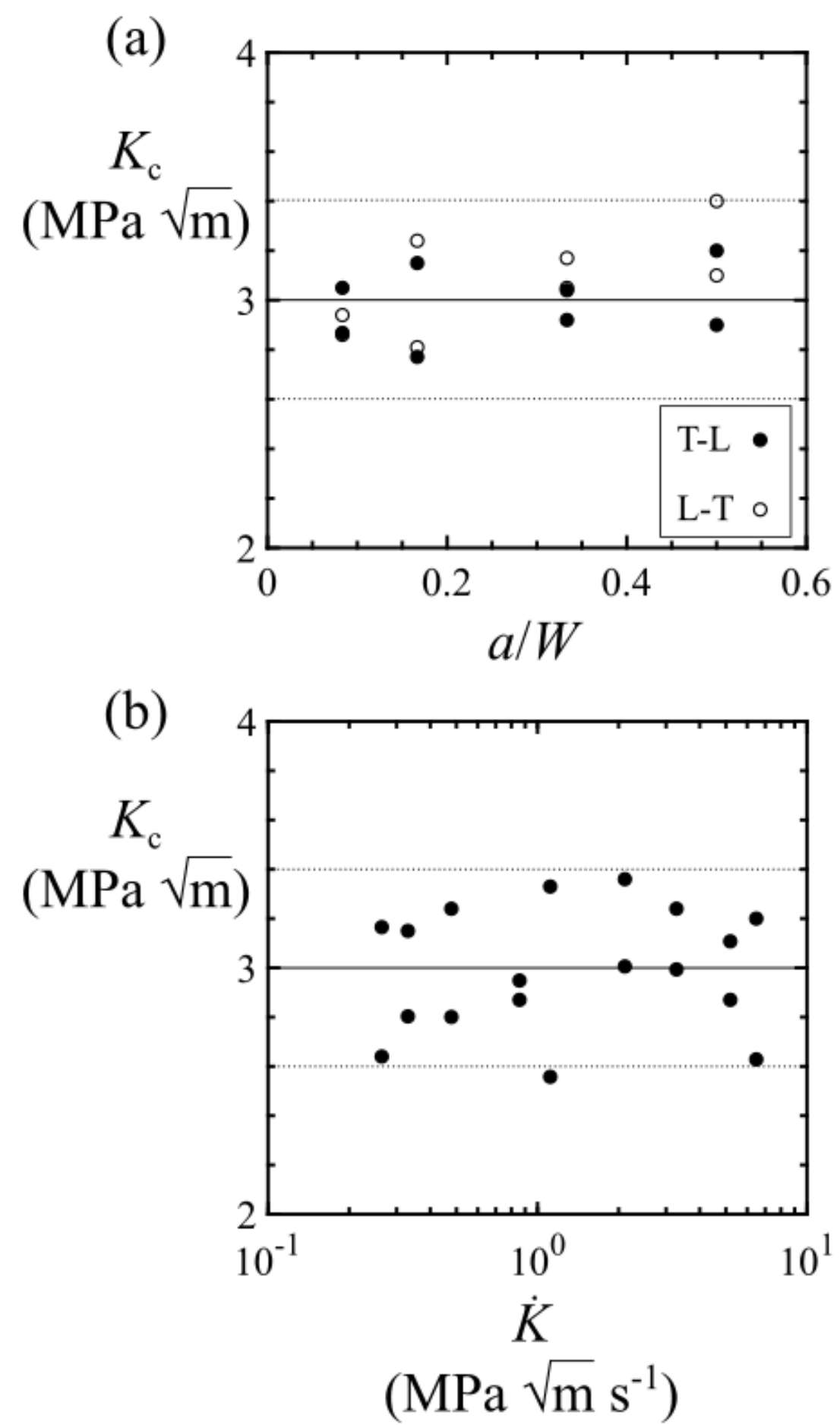

Figure 13 Plane-stress, fracture-toughness measurements for the cellulose acetate tape: (a) $K_{\mathrm{c}}$ versus $a / W$, for the geometries shown in Figure 11c and 11d; (b) $K_{\mathrm{c}}$ versus $\dot{K}$. Crack length $a$ is measured to an accuracy of $0.2 \mathrm{~mm}$. The mean and range of fracture toughness values are included in each figure as a solid line and dotted lines, respectively. 


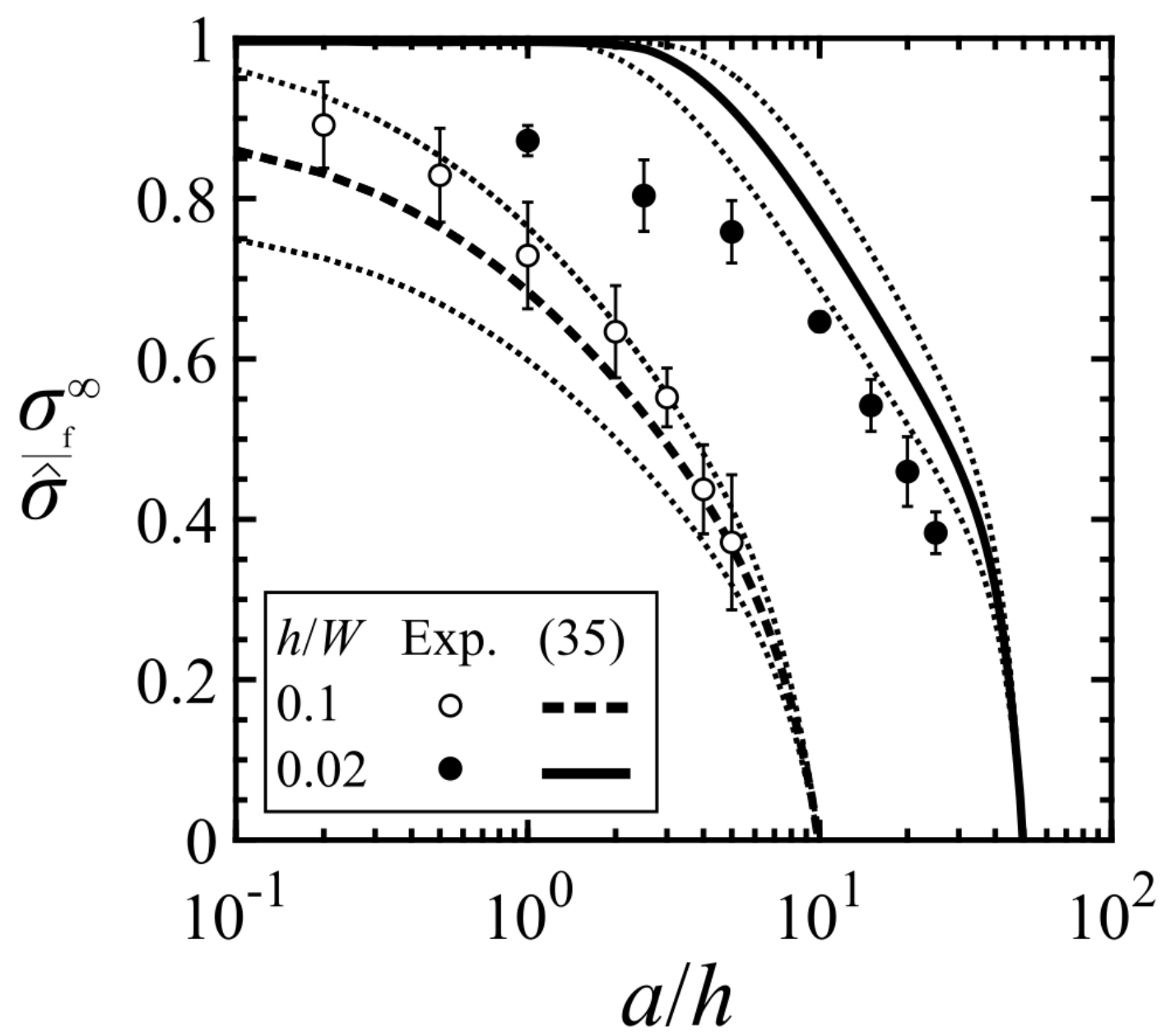

Figure 14 Measured and predicted strength $\sigma_{\mathrm{f}}^{\infty} / \hat{\sigma}$ of sandwich specimens versus $a / h$ for $h / W=0.1$ and $h / W=0.02$. The values of $l_{\mathrm{S}} / h$ are 0.2 and 1 for $h / W$ equal to 0.1 and 0.02 , respectively. 


\section{Appendix A: A semi-infinite crack in a sandwich layer}

The stress state ahead of the tip of a semi-infinite crack in a sandwich layer in a state of plane stress, and subjected to a remote stress intensity factor $K^{\infty}$, is determined by finite element (FE) calculations using the implicit solver of ABAQUS (version 6.14). Recall that the crack geometry, absent a cohesive zone, has already been defined in Figure 2. The displacement field associated with a remote mode-I $K$ - field of magnitude $K^{\infty}$ is applied on a semi-circular outer boundary of radius $R$ enclosing the crack tip (Irwin, 1957; Anderson, 2005) as specified by

$$
u_{x x}=\frac{2\left(1+v_{1}\right) K^{\infty}}{E_{1}}\left(\frac{r}{2 \pi}\right)^{\frac{1}{2}} \cos \left(\frac{\theta}{2}\right)\left[1-2 v_{1}+\sin ^{2}\left(\frac{\theta}{2}\right)\right]
$$

and

$$
u_{y y}=\frac{2\left(1+v_{1}\right) K^{\infty}}{E_{1}}\left(\frac{r}{2 \pi}\right)^{\frac{1}{2}} \sin \left(\frac{\theta}{2}\right)\left[2-2 v_{1}-\cos ^{2}\left(\frac{\theta}{2}\right)\right]
$$

where $\boldsymbol{u}_{\boldsymbol{x}}$ and $\boldsymbol{u}_{\boldsymbol{y}}$ are the horizontal and vertical displacements of the nodes of the semi-circular boundary in terms of polar $(r, \theta)$ coordinates, and plane strain conditions are assumed. The substrate and the adhesive layer are discretised by 8-noded bi-quadratic plane strain elements with reduced integration (CPE8R), and quarter-point elements are used at the crack tip. We take $R=180000 h$, and the total number of elements is approximately 10000 . The Young's modulus of the substrate material $E_{1}$ is held fixed while a range of values are assumed for the Young's modulus of the adhesive layer; the Poisson's ratios of substrate and layer are held fixed at $v=v_{1}=v_{2}=0.3$, implying that $\bar{v}=v /(1-v)=3 / 7$. The predicted distribution of the tensile stress component $\sigma_{y y}$ along the crack is given in Figure 3.

\section{Appendix B: Finite element validation of the analytical theory}

Two dimensional finite FE simulations are performed using the implicit solver of ABAQUS (version 6.14). First, the macroscopic strength of the sandwich layer shown in Figure 1 is computed as a function of crack length for the case where the cohesive zone at the crack tip is absent, $l_{S}=0$. The effect of modulus mismatch and adhesive layer thickness on the 
strength of the joint is explored. Second, a layer of tensile cohesive elements is added directly ahead of the crack tip.

\section{B.1 Finite element simulations for a crack in an elastic sandwich layer absent a cohesive zone}

We model the sandwich layer by treating both the adhesive and the substrate as isotropic, elastic solids. The length of the cohesive zone $c$ equals zero, see Figure 1. Simulations are performed for normalized crack lengths ranging from $a / h=0.1$ to $a / h=30$, with $h / W=10^{-1}$ and $h / W=10^{-2}$. The ratio of Young's modulus of the adhesive $E_{2}$ to that of the substrate ranges from $10^{-3}$ to 0.1 , with $v=v_{1}=v_{2}=0.3$. Only a quarter of the sandwich specimen is modeled due to the symmetry of the problem. The substrate and the adhesive layer are discretized by 8-noded bi-quadratic plane strain elements (CPE8). A quarter point element is located at the crack tip and the mesh is refined close to the crack tip. The total number of elements is a function of the crack half-length $a$ and lies in the range of 100000 to 200000 . A finite value of remote tensile stress $\sigma^{\infty}$ is applied, and the $J$-integral is evaluated at the crack tip. Upon writing $\Gamma=J$ and $\sigma^{\infty}=\sigma_{f}^{\infty}$, the predicted normalized strength $\bar{\sigma}$ is obtained and plotted as a function of normalized crack length $a / h$, see Figure B1a for a modulus mismatch ratio $E_{2} / E_{1}=10^{-1}$. The analytical predictions (10) for the adhesive-governed strength regime and (23) for the substrate-governed strength regime are included in Figure B1a, and good agreement is noted between the FE predictions and the analytical theory. Since the FE analysis assumes plane strain, with $v=0.3$, the appropriate values for $\bar{v}$ and $\bar{E}_{2} / \bar{E}_{1}$ in the analytical model are given by $\bar{v}=3 / 7$ and $\bar{E}_{2} / \bar{E}_{1}=E_{2} / E_{1}$. Equation (23) slightly overestimates $\bar{\sigma}$ as obtained by the FE simulations in the regime where the crack length is comparable to the adhesive layer height. Recall that the prediction (12) for a long crack $(a / h>>1)$ neglects the presence of the adhesive layer in the Irwin relation (9). This asymptote is included in Figure B1a: the formula (23) converges to (12) for $a / h>10$.

The computed $\bar{\sigma}$ versus $a / h$ characteristic, as obtained by FE analysis, is plotted in Figure B1b for $E_{2} / E_{1}=10^{-2}$ and in Figure B1c for $E_{2} / E_{1}=10^{-3}$. The predictions (10) for the adhesive-governed strength regime and (23) for the substrate-governed strength regime are included in both figures. Excellent agreement is noted between the predicted values for $\bar{\sigma}$ by the analytical theory and by the FE model for the explored range of $a / h$ values. Recall that 
the remote $K$-field, $K^{\infty}$, only exists for a sufficient long crack, as discussed in relation to (4). The long-crack asymptote (12) has been added to Figures B1b and B1c, and it is expected to align with the prediction (23) for values $a / h$ beyond the range plotted.

\section{B.2 Finite element simulations for a crack in an elastic sandwich layer with a cohesive zone}

The strength of the sandwich specimen, as defined in Figure 1, has also been computed by accounting for the presence of a cohesive zone at the crack tip. A layer of tensile cohesive elements is now included in the FE model of the previous section. The tensile cohesive zone extends along the $x$-axis from the crack tip to the free surface at the right-hand side of the sandwich layer. The traction $T$ versus separation $\delta$ law of the cohesive elements is linear with slope $k$ until $T$ attains the cohesive strength $\hat{\sigma}$, beyond which $T=\hat{\sigma}$. We treat the adhesive and the substrate as isotropic, homogeneous and linear, elastic materials. Simulations are performed for normalized crack lengths ranging from $a / h=0.03$ to $a / h=30$, for $h / W=10^{-1}$ and for $h / W=10^{-2}$. As for the FE simulations absent a cohesive zone, the Young's modulus of the substrate material $E_{1}$ is held fixed while a range of values are assumed for the Young's modulus of the adhesive layer; the Poisson's ratios of substrate and layer are held fixed at $v=v_{1}=v_{2}=0.3$.

The substrate and the adhesive layer are meshed by 8-noded bi-quadratic plane strain elements (CPE8), and 4-noded cohesive element (COH2D4) are used for the cohesive elements. Define $l_{\mathrm{e}}$ as the characteristic cohesive element size. Then, for an accurate numerical solution, we require $l_{\mathrm{e}} k / E_{2}>>1$. Additionally, $l_{\mathrm{e}}$ has to be sufficiently small to resolve the cohesive zone at the crack tip: $l_{\mathrm{e}} / l_{\mathrm{S}} \leq 1$ (del Busto et al., 2017). A mesh sensitivity study led to the choice $l_{\mathrm{e}} k / E_{2}=100$. The total number of elements depends upon the choice of $a$ and lies between 150000 and 250000 .

For each simulated geometry, the remote tensile stress $\sigma^{\infty}$ is incremented in the range $\sigma^{\infty} / \hat{\sigma}=0.1$ to $\sigma^{\infty} / \hat{\sigma}=0.99$, and the associated value of cohesive zone opening at the crack tip $\delta^{\text {tip }}$ is determined. Upon writing $\sigma^{\infty}=\sigma_{\mathrm{f}}^{\infty}$, the toughness $\Gamma$ corresponding to any imposed value of $\sigma^{\infty}$ is obtained by 


$$
\Gamma=\hat{\sigma} \delta^{\text {tip }}-\frac{\hat{\sigma}^{2}}{2 k}
$$

and thereby the value of $l_{\mathrm{S}}$ via (6). The FE predictions are presented in the form of maps with axes $\left(a / h, l_{\mathrm{S}} / h\right)$ for a modulus mismatch ratio $E_{2} / E_{1}$ of $10^{-1}, 10^{-2}$ and $10^{-3}$, in Figures B2a, B2b and B2c, respectively. In each plot, contours of $\sigma^{\infty} / \hat{\sigma}$ are presented for $h / W=10^{-1}$ and $h / W=10^{-2}$, and the analytical formulae (27) and (36) are included, along with the active failure regime, as implied by the analytical formulae. The analytical predictions are in close alignment with the FE results. 
Figures Appendix

\begin{tabular}{|cc|}
\hline$h / W$ & $\mathrm{FE}$ \\
$10^{-1}$ & $\circ$ \\
$10^{-2}$ & $\bullet$ \\
\hline
\end{tabular}

(a)

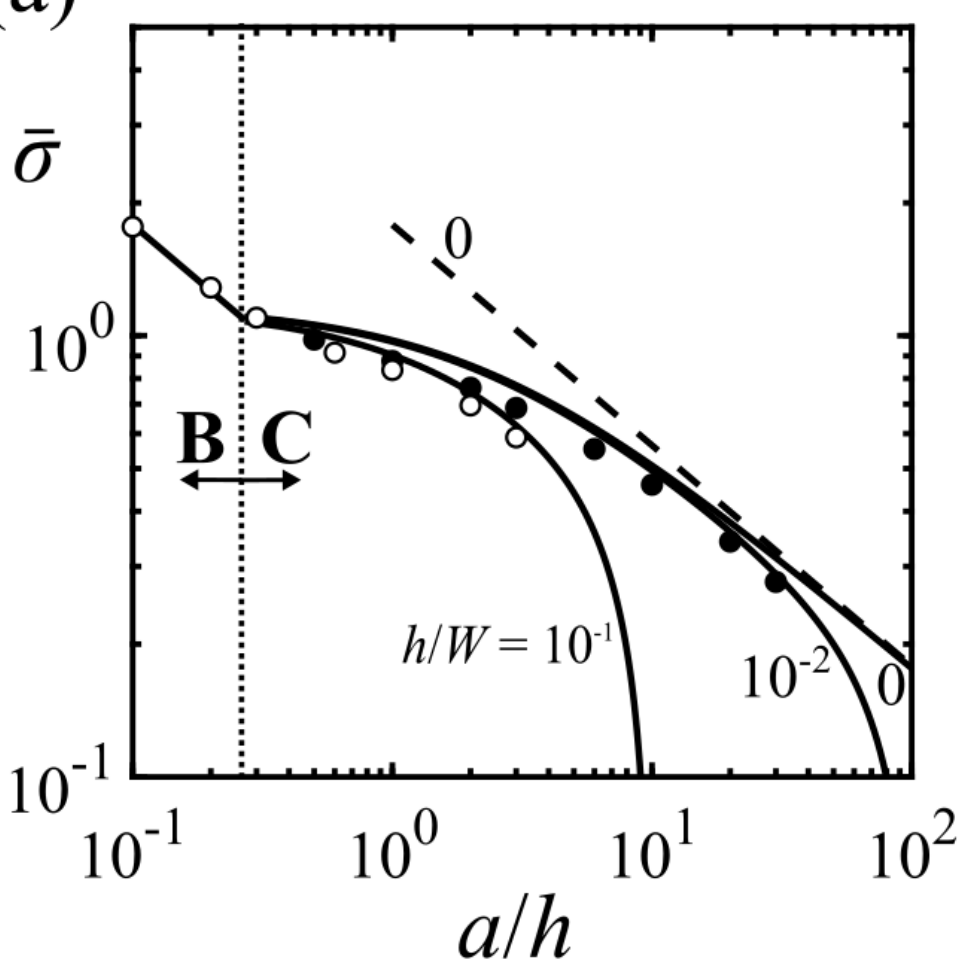

(b)

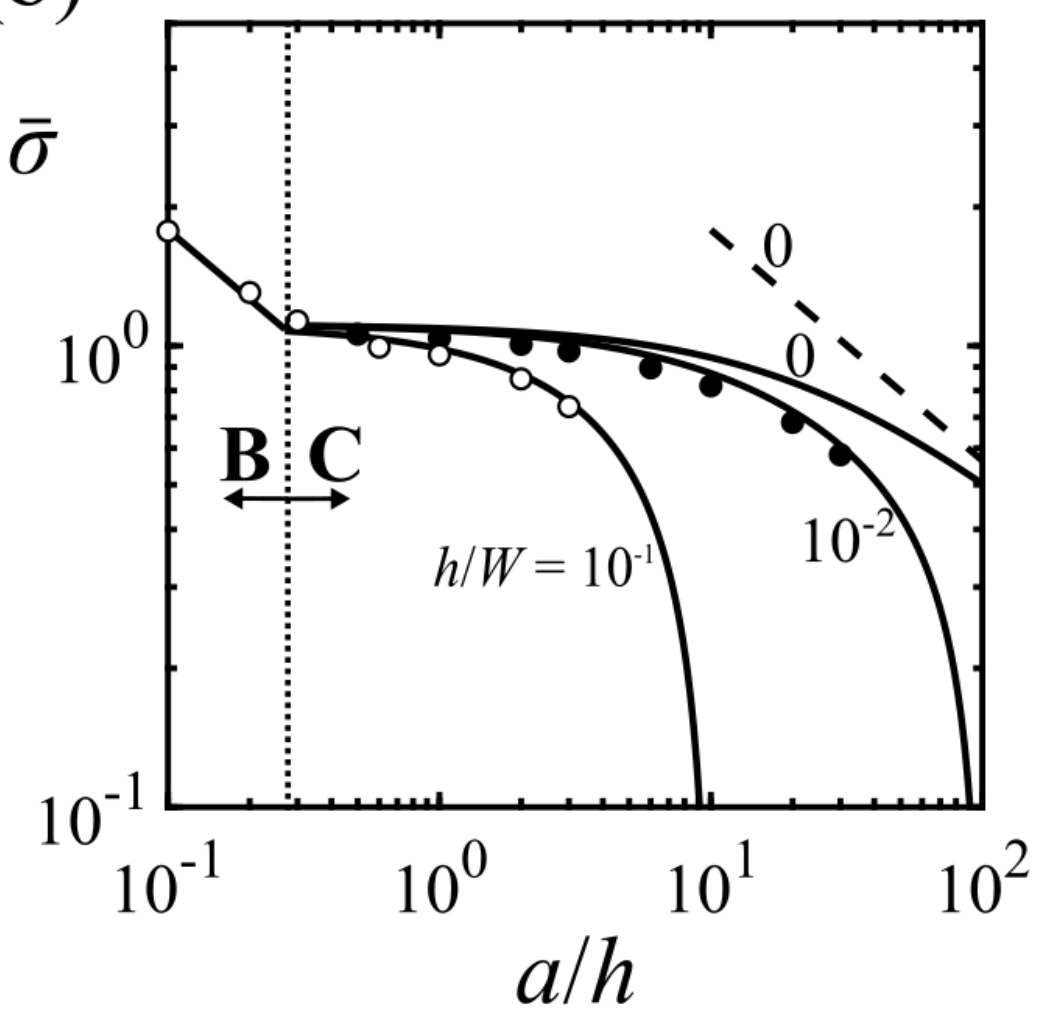




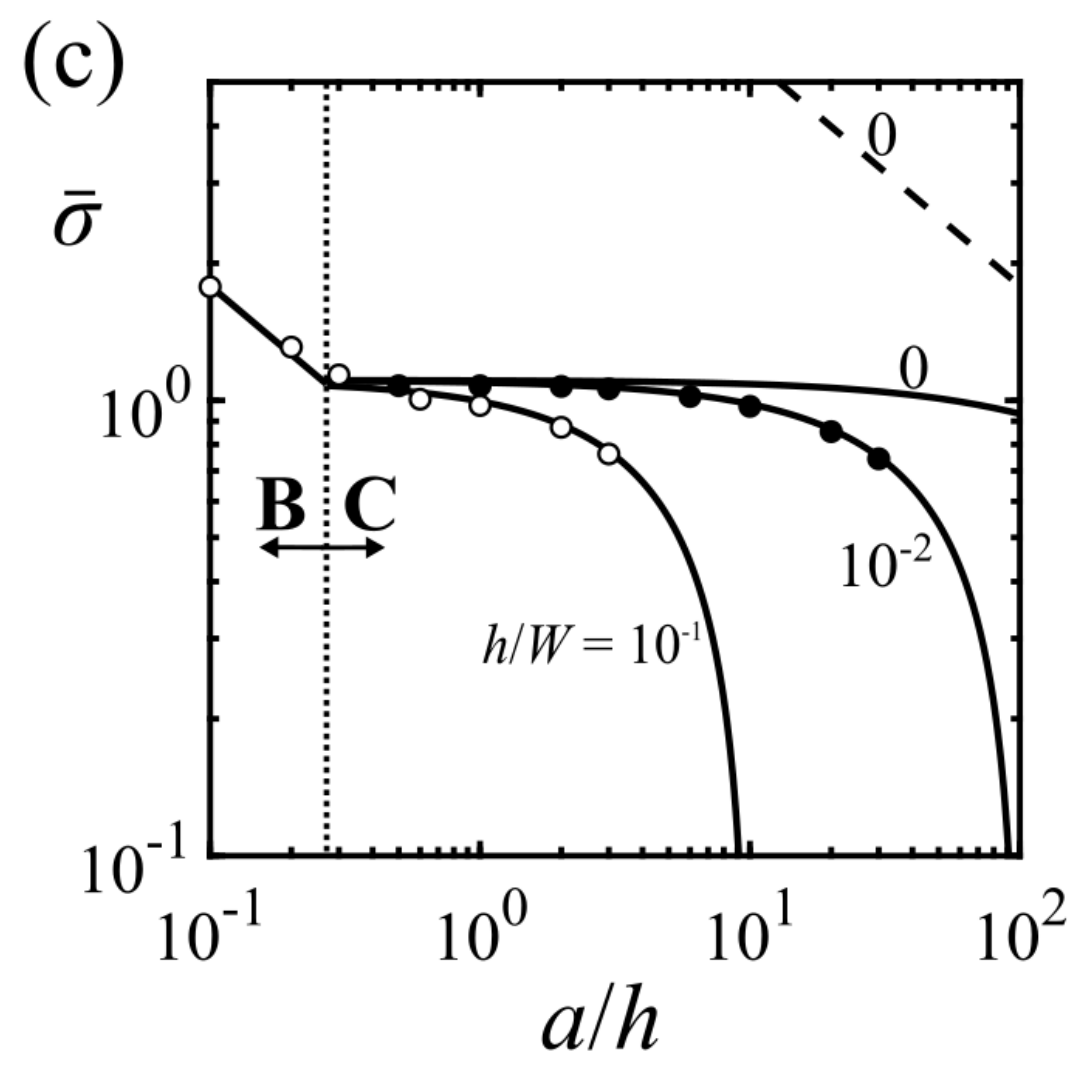

Figure B1 Predicted $\bar{\sigma}$ versus $a / h$ data by the FE model for $h / W=10^{-1}$ and $h / W=10^{-2}$ for (a) $E_{2} / E_{1}=10^{-1}$; (b) $E_{2} / E_{1}=10^{-2}$; and (c) $E_{2} / E_{1}=10^{-3}$. In regime $B$, the strength is predicted by (10). In regime $C$, the strength is predicted from (23). The dotted asymptotic limit lines in regime $\mathrm{C}$ are given by (12). 


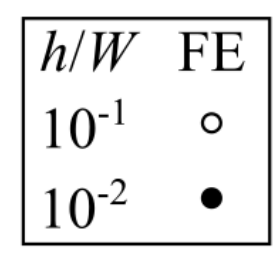

(a)

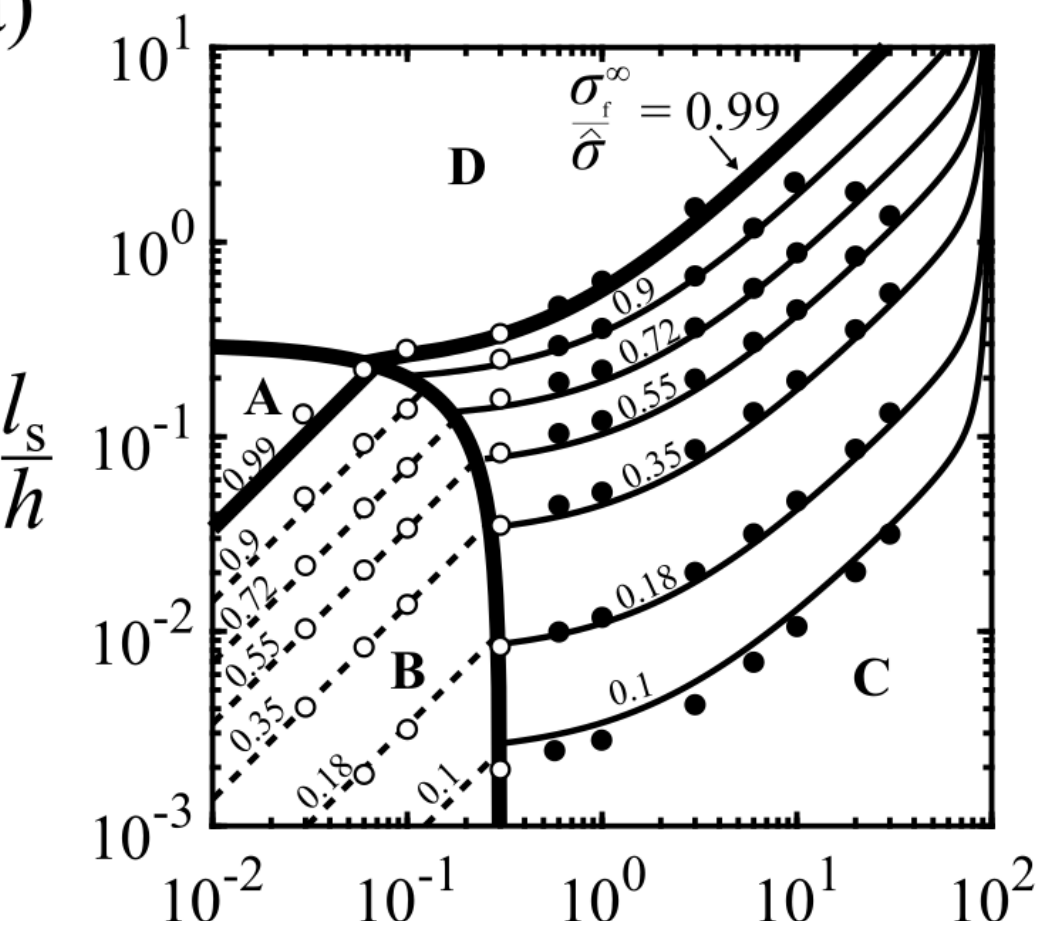

(b)

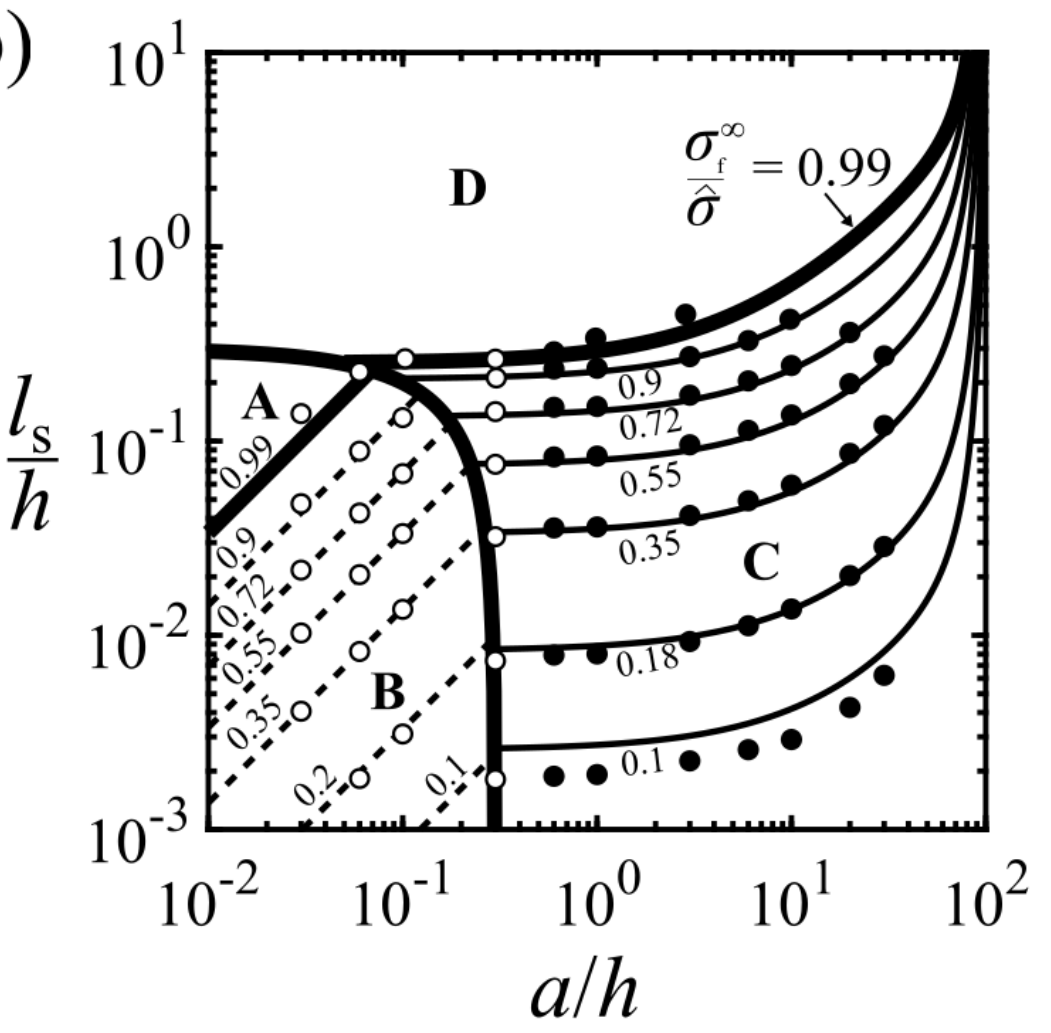




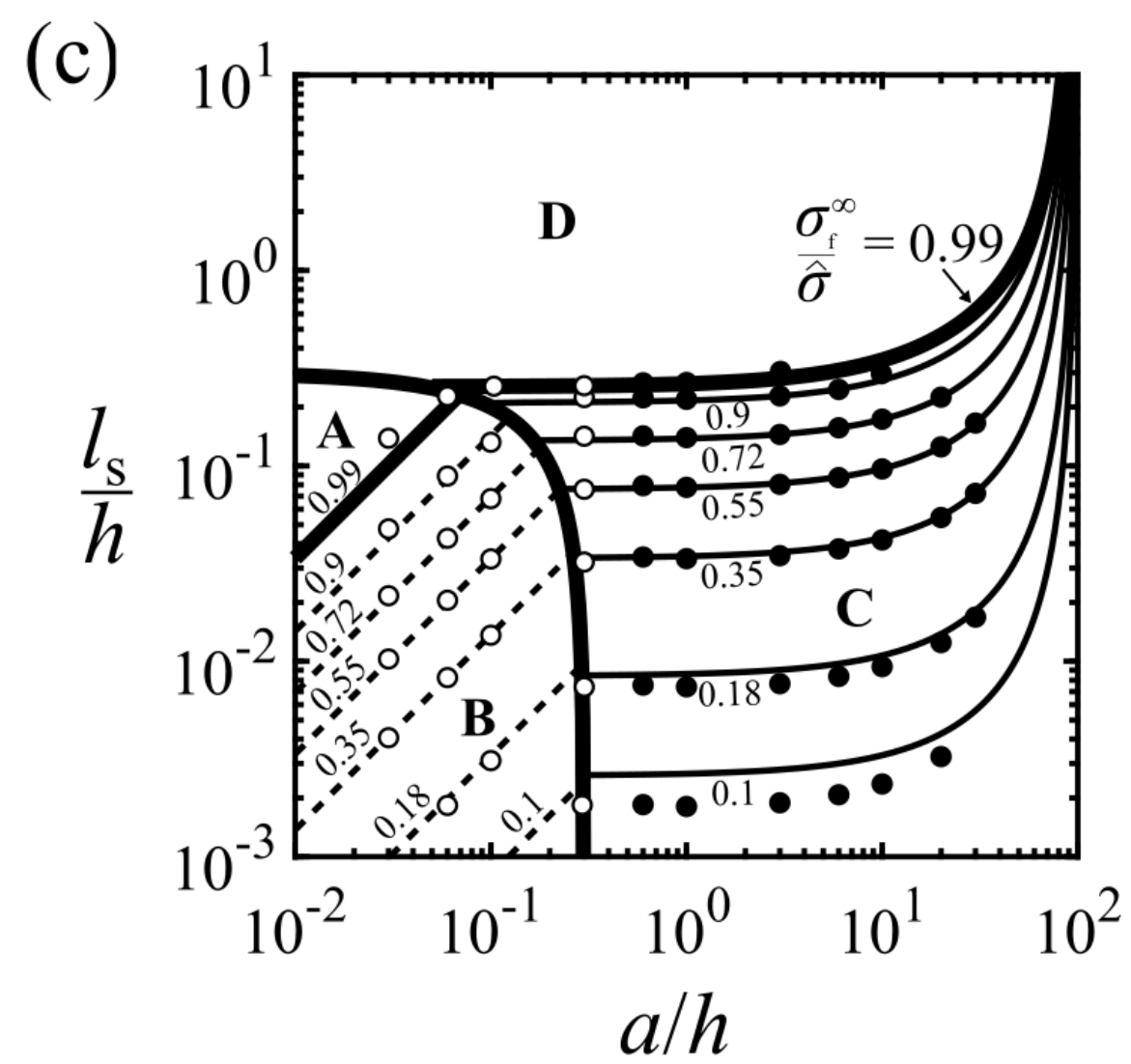

Figure B2 Failure map of $l_{\mathrm{S}} / \mathrm{h}$ versus normalized crack length $a / h$ for $h / W=10^{-2}$ and $h / W=10^{-1}$. (a) $E_{2} / E_{1}=10^{-1}$; (b) $E_{2} / E_{1}=10^{-2}$; and (c) $E_{2} / E_{1}=10^{-3}$. 


\section{References}

Banea, M.D., Da Silva, L.F.M., Campilho, R.D.S.G., 2014. The effect of adhesive thickness on the mechanical behavior of a structural polyurethane adhesive. J. Adhes. 91, 331346. https://doi.org/10.1080/00218464.2014.903802

Barenblatt, G.I., 1962. The Mathematical Theory of Equilibrium Cracks in Brittle Fracture. Adv. Appl. Mech. 7, 55-129. https://doi.org/https://doi.org/10.1016/S00652156(08)70121-2

Bascom, W.D., Cottington, R.L., Jones, R.L., Peyser, P., 1975. The fracture of epoxy- and elastomer-modified epoxy polymers in bulk and as adhesives. J. Appl. Polym. Sci. 19, 2545-2562. https://doi.org/10.1002/app.1975.070190917

Benthem, J.P., Koiter, W.T., 1972. Asymptotic approximations to crack problems, in: Sih, G.C. (Ed.), Methods of Analysis of Crack Problems. Noordhoff International Publishing, pp. 131-178.

Blackman, B.R.K., Hadavinia, H., Kinloch, A.J., Paraschi, M., Williams, J.G., 2003. The calculation of adhesive fracture energies in mode I: Revisiting the tapered double cantilever beam (TDCB) test. Eng. Fract. Mech. 70, 233-248. https://doi.org/10.1016/S0013-7944(02)00031-0

Bonaldo, J., Banea, M.D., Carbas, R.J.C., Da Silva, L.F.M., De Barros, S., 2018. Functionally graded adhesive joints by using thermally expandable particles. J. Adhes. https://doi.org/10.1080/00218464.2018.1456338

Camanho, P., Tong, L., 2011. Composite joints and connections : principles, modelling and testing. Woodhead Pub.

Camanho, P.P., Davila, C.G., Pinho, S.S., 2004. Fracture analysis of composite co-cured structural joints using decohesion elements. Fatigue Fract. Eng. Mater. Struct. 27, 745757. https://doi.org/10.1111/j.1460-2695.2004.00695.x

Carlberger, T., Stigh, U., 2010. Influence of layer thickness on cohesive properties of an epoxy-based adhesive-an experimental study. J. Adhes. 86, 816-835. https://doi.org/10.1080/00218464.2010.498718

Chevalier, J., Morelle, X.P., Bailly, C., Camanho, P.P., Pardoen, T., Lani, F., 2016. Micromechanics based pressure dependent failure model for highly cross-linked epoxy resins. Eng. Fract. Mech. 158, 1-12. https://doi.org/10.1016/j.engfracmech.2016.02.039

da Silva, L., Ochsner, A., Adams, R., 2018. Handbook of Adhesion Technology, Second Edi. ed. Springer.

del Busto, S., Betegón, C., Martínez-Pañeda, E., 2017. A cohesive zone framework for environmentally assisted fatigue. Eng. Fract. Mech. 185, 210-226. https://doi.org/10.1016/j.engfracmech.2017.05.021

Dillard, D.A., 2010. Advances in structural adhesive bonding. Woodhead Pub. https://doi.org/10.1533/9781845698058

Du, J., Thouless, M.D., Yee, A.F., 1998. Development of a process zone in rubber-modified epoxy polymers. Int. J. Fract. 92, 271-286.

Dugdale, D.S., 1960. Yielding of steel sheets containing slits. J. Mech. Phys. Solids 8, 100- 
104. https://doi.org/10.1016/0022-5096(60)90013-2

Federsen, C.E., 1966. Discussion to `Plane strain crack toughness testing’ ASTM Special Technical Publication.

Fiedler, B., Hojo, M., Ochiai, S., Schulte, K., Ochi, M., 2001. Finite-element modeling of initial matrix failure in CFRP under static transverse tensile load. Compos. Sci. Technol. 61, 1615-1624. https://doi.org/10.1016/S0266-3538(00)00198-6

Fleck, N.A., Hutchinson, J.W., Suo, Z., 1991. Crack path selection in a brittle adhesive layer. Int. J. Solids Struct. 27, 1683-1703. https://doi.org/10.1016/0020-7683(91)90069-R

Higgins, A., 2000. Adhesive bonding of aircraft structures. Int. J. Adhes. Adhes. 20, 367376. https://doi.org/10.1016/S0143-7496(00)00006-3

Hui, C.Y., Ruina, A., Creton, C., Kramer, E.J., 1992. Micromechanics of Crack Growth into a Craze in a Polymer Glass. Macromolecules 25, 3948-3955. https://doi.org/10.1021/ma00041a018

Imanaka, M., Ikeda, K., Nakamura, Y., Kimoto, M., 2015. Fracture behaviour of epoxy resins modified with liquid rubber and crosslinked rubber particles under mode I loading. Polym. Polym. Compos. 23, 399-406. https://doi.org/10.1016/j.ijadhadh.2007.11.004

Knauss, W.G., 1966. Stresses in an Infinite Strip Containing a Semi-Infinite Crack. J. Appl. Mech. 33, 356-362.

Lee, D.-B., Ikeda, T., Miyazaki, N., Choi, N.-S., 2004. Effect of Bond Thickness on the Fracture Toughness of Adhesive Joints. J. Eng. Mater. Technol. 126, 14-18. https://doi.org/10.1115/1.1631433

Li, S., Thouless, M.D., Waas, A.M., Schroeder, J.A., Zavattieri, P.D., 2006. Mixed-mode cohesive-zone models for fracture of an adhesively bonded polymer-matrix composite. Eng. Fract. Mech. 73, 64-78. https://doi.org/10.1016/j.engfracmech.2005.07.004

Lopes, R.M., Campilho, R.D.S.G., Da Silva, F.J.G., Faneco, T.M.S., 2016. Comparative evaluation of the Double-Cantilever Beam and Tapered Double-Cantilever Beam tests for estimation of the tensile fracture toughness of adhesive joints. Int. J. Adhes. Adhes. 67, 103-111. https://doi.org/10.1016/j.ijadhadh.2015.12.032

Martiny, P., Kinloch, F.L.A.J., Pardoen, T., 2012. A multiscale parametric study of mode I fracture in metal-to-metal low-toughness adhesive joints. Int. J. Fract. 173, 105-133. https://doi.org/10.1007/s10704-011-9667-x

Monteiro, J.P.R., Campilho, R.D.S.G., Marques, E.A.S., da Silva, L.F.M., 2015. Experimental estimation of the mechanical and fracture properties of a new epoxy adhesive. Appl. Adhes. Sci. 3, 1-17. https://doi.org/10.1186/s40563-015-0056-y

Pandya, K.C., Ivankovic, A., Williams, J.G., 2000. Cohesive zone modelling of crack growth in polymers Part 2 - Numerical simulation of crack growth. Composites 29, 447-452. https://doi.org/10.1179/146580100101541283

Pardoen, T., Ferracin, T., Landis, C.M., Delannay, F., 2005. Constraint effects in adhesive joint fracture. J. Mech. Phys. Solids 53, 1951-1983.

https://doi.org/10.1016/j.jmps.2005.04.009

Rice, J.R., 1968. “A Path Independent Integral and the Approximate Analysis of Strain 
Concentration by Notches and Cracks.” J. Appl. Mech. 35, 379.

https://doi.org/10.1115/1.3601206

Rice, J.R., 1967. Discussion: “Stresses in an infinite strip containing a semi-infinite crack" (Knauss, W. G., 1966, ASME J. Appl. Mech., 33, pp. 356-362). J. Appl. Mech. 34, 248-250.

Ryvkin, M., 2000. K -Dominance zone for a semi-infinite mode I crack in a sandwich composite. Int. J. Solids Struct. 37, 4825-4840.

Salomonsson, K., Andersson, T., 2008. Modeling and parameter calibration of an adhesive layer at the meso level. Mech. Mater. 40, 48-65.

https://doi.org/10.1016/j.mechmat.2007.06.004

Schellekens, J.C.J., de Borst, R., 1996. On the numerical modelling of edge delamination in composites. Key Eng. Mater. 120-121, 131-160.

Stigh, U., Alfredsson, K.S., Andersson, T., Biel, A., Carlberger, T., Salomonsson, K., 2010. Some aspects of cohesive models and modelling with special application to strength of adhesive layers. Int. J. Fract. 165, 149-162. https://doi.org/10.1007/s10704-010-9458-9

Sun, C., Thouless, M.D., Waas, A.M., Schroeder, J.A., Zavattieri, P.D., 2008. Ductile-brittle transitions in the fracture of plastically deforming, adhesively bonded structures. Part II: Numerical studies. Int. J. Solids Struct. 45, 4725-4738. https://doi.org/10.1016/j.ijsolstr.2008.04.007

Tada, H., Paris, P., Irwin, G., 2000. The Stress Analysis of Cracks Handbook, Third Edit. ed. The American Society of Mechanical Engineers. https://doi.org/10.1115/1.801535

Thouless, M.D., Waas, A.M., Schroeder, J.A., Zavattieri, P.D., Sun, C., 2008. Ductile-brittle transitions in the fracture of plastically-deforming, adhesively-bonded structures. Part I: Experimental studies. Int. J. Solids Struct. 45, 3059-3073. https://doi.org/10.1016/j.ijsolstr.2008.01.011

Trantina, G.G., 1972. Fracture mechanics approach to adhesive joints. J. Compos. Mater. 6, 192-207.

Tsou, A.H., Greener, J., Smith, G.D., 1995. Stress relaxation of polymer films in bending. Polymer (Guildf). 36, 949-954. https://doi.org/10.1016/0032-3861(95)93593-B

Tvergaard, V., Hutchinson, J.W., 1996. On the toughness of ductile adhesive joints. J. Mech. Phys. Solids 44, 789-800.

Tvergaard, V., Hutchinson, J.W., 1994. Toughness of an interface along a thin ductile layer joining elastic solids. Philos. Mag. A 70, 641-656.

https://doi.org/10.1080/01418619408242253

Tvergaard, V., Hutchinson, J.W., 1992. The relation between crack growth resistance and fracture process parameters in elastic-plastic solids. J. Mech. Phys. Solids 40, 13771397. https://doi.org/10.1016/0022-5096(92)90020-3

Varias, A.G., Suo, Z., Shih, C.F., 1991. Ductile failure of a constrained metal foil. J. mech 39, 963-986. https://doi.org/https://doi.org/10.1016/0022-5096(91)90014-F

Wang, C.H., 1997. Analysis of cracks in constrained layers. Int. J. Fract. 83, 1-17. https://doi.org/10.1023/A:1007313620138 
Wang, S.S., Mandell, J.F., McGarry, F.J., 1978. An analysis of the crack tip stress field in DCB adhesive fracture specimens. Int. J. Fract. 14, 39-58.

https://doi.org/10.1007/BF00032383

Williams, M.L., 1957. On the stress distribution at the base of a stationary crack. J. Appl. Mech. 24, 109-114. https://doi.org/10.1115/1.3640470

Yang, Q., Cox, B., 2005. Cohesive models for damage evolution in laminated composites. Int. J. Fract. 133, 107-137. https://doi.org/10.1007/s10704-005-4729-6 